\title{
On how proton radius shrinkage can be connected with Lorentz factor violation
}

\author{
Wladimir Guglinski \\ Escola de Engenharia da Universidade Federal de Minas Gerais, Av. Presidente Antonio Carlos, 6627, \\ Pampulha, Belo Horizonte-MG, Brazil
}

E-mail addresses: wladimirguglinski@hotmail.com

Received Feb 2017

Received in revised: March 2018

Published : June 2018

\begin{abstract}
ABSTRA CT
Several new experimental findings have shown that atomic nuclei cannot have similar structure of that adopted in the Standard Nuclear Physics (SNP), because there are insurmountable obstacles to be transposed. Nuclear theorists have tried to explain some of the misfires with bizarre theories, but there is a failure impossible to be explained by any theoretical attempt, and such failure impossible to be solved represents the definitive proof that SNP works through wrong foundations. The failure comes from the excited isotopes carbon-12, oxygen-16, argon-36, calcium-40, and calcium-42. All them with spin 2 , have null magnetic moments, but this is impossible, because it's any combination of spins from which those excited isotopes, with spin 2 , may have null magnetic moment, if we try to explain it with any of the current nuclear models of the SNP. And the unavoidable conclusion is that it's impossible to eliminate the inconsistences of the SNP by keeping its current fundamental premises.
\end{abstract}

Keywords: New nuclear model, Ellipsoidal even-even nuclei, Electron \& positron substructures, Nuclear puzzles

C2018 The Authors. Published by Fundamental Journals. This is an open access article under the CC BY-NC https://creativecommons.org/licenses/by-nc/4.0/

https://doi.org/10.14331/ijfps.2018.330114

\section{INTRODUCTION}

An atomic nucleus with $Z$ and $N$ pairs, excited with spin +2 , cannot have null nuclear magnetic moment, because it is impossible any combination of spins capable to generate a null magnetic moment when the atomic nucleus has non-null spin.
But there are several isotopes with $Z$ and $N$ pairs (some of them with $Z=N$ ), excited with spin +2 , whose magnetic moments are not quoted in nuclear tables. They are as, 6C12, 8016, 12Mg24, 14Si32, 18Ar36, 20Ca40, 20Ca42, 24Cr48, 26Fe52, $28 \mathrm{Ni56.}$ 


\section{Implication}

Null magnetic moments for those excited isotopes implies that the current Nuclear Theory is definitively wrong. So, how do the nuclear physicists deal with such puzzle? There are two hypotheses to be considered.

A-Their magnetic moments were never measured. This is the argument used by nuclear theorists, in special the editors of the most reputable journals of physics. The editors claim that those excited isotopes have non null magnetic moment, but as the experimentalists have never measured them, this is the reason why their magnetic moments are not quoted in nuclear tables. This is the way the Editors-in-chief of the most reputable journals of physics avoid the definitive breakdown of the Nuclear Physics.

B-Their magnetic moments were measured, but as the experimentalists found values zero, they did not report their measurements for the editors of nuclear tables.

\section{Analysis of hypothesis A}

The hypothesis $A$ is used by editors of reputable journals, but it is denied by the fact that many of those excited isotopes have their electric quadrupole moments quoted in nuclear tables. They are (in barns), 6C12, $Q=+0.06,12 \mathrm{Mg} 24, Q=-0.29$, 14 Si32, $Q=-0.16,18 \operatorname{Ar} 36, Q=+0.11,20 \operatorname{Ca} 42, Q=-0.19$.

\section{Analysis of hypothesis B}

When the experimentalists have measured the electric quadrupole moments for the excited $6 C 12,12 \mathrm{Mg} 24,14 \mathrm{Si32}$, 18Ar36, and 20Ca42, of course they have also measured their magnetic moment, because all experimentalists aim to provide data for constructing a complete nuclear table, with all (measurable) nuclear properties of all isotopes of the whole elements of the Periodic Table.

\section{Conclusion of the hypothesis B}

Therefore, it is discarded the hypothesis that the experimentalists did not measure the magnetic moment for the excited $6 C 12,12 \mathrm{Mg} 24,14 \operatorname{Si} 32,18 \mathrm{Ar} 36$, and $20 \mathrm{Ca} 42$, because it makes no sense to suppose that they have measured the electric quadrupole moments, but the magnetic moments they did not do (it makes no sense because to measure magnetic moment is easier than to measure electric quadrupole moment).

\section{INEVITABLE CONCLUSIONS}

1. The experimentalists have measured the magnetic moments of those excited isotopes.

2. They did not report their results, for the editors of nuclear tables, because the magnetic moment measured, for all those nuclei, was ZERO.

3. It seems the editors of nuclear tables have adopted the strategy of do not quote zero the magnetic moments when the experiments do not detect any value different of zero. By this way they avoid to quote "zero" the magnetic moments of the several nuclei with $Z$ and $N$ pairs, excited with spin +2 , because to quote them zero would imply in the breakdown of the Nuclear Theory.

All the current nuclear models (in which protons and neutrons are bound via strong nuclear force) are wrong, because there is not any of them capable to explain why the excited $6 C 12,12 \mathrm{Mg} 24,14 \operatorname{Si32}, 18 \mathrm{Ar} 36$, and 20Ca42, have null magnetic moment.

\section{THE ROLE PLAYED BY SPECULATIONS IN THE DEVELOPMEN OF THE THEORETICAL PHYSICS}

Theorists would like the development of physical theories could be successful via a process of investigation free of speculations, and this is a healthy aim, preconized by great scientists as Newton and Einstein. But Einstein, face to the crisis opened by the result of the Michelson-Morley experiment, understood that the aim was unattainable, and he was constrained to adopt several speculations. In the $20^{\text {th }}$ Century the situation became worst, because besides the proposal of speculations along the development of the theories (quantum mechanics, nuclear theory, and particles physics), some of them were very strange and even nonsensical, because (as the contribution of the aether was neglected in the development of the theories), it was impossible to develop the theories with "reasonable" speculations, inasmuch the missing of the contribution of the aether in the theories had led the theorists to the conclusion that nature has no logic, and therefore crazy speculations have to be adopted. For instance, Heisenberg proposed a nonsensical speculation, the isospin, because instead of explaining what is the force which avoids the formation of two neutrons bound through the strong force, the explanation by the isospin means that such a force is created by an abstract mathematical concept, and it is obvious that the Mathematics cannot create forces so that to apart neutrons, and the unavoidable consequence would be to conclude that something was wrong with the concept of interaction though the strong force. The situation today is similar to that faced by Galileo, when he understood that Aristotle failed in his hypothesis that heavy body fall down quickly than the light ones, because he neglected to consider the contribution of the air in the mechanism of the fall of the bodies. The error of the theorists today is to be sure that they are very smart, and there is no way they may commit the same error committed by Aristotle, because they have today at hand a fantastic tool not available for the greek philosopher, the Mathematics. But the Mathematics is no magic, and, if used in the wrong way, she cannot produce miracles, as to create forces which separate two neutrons bound via the strong force. The situation of the theorists is actually worst, because they are sure that the Mathematics is leading them in the correct way, but the reality is other, since the Mathematics applied in the wrong way creates a fatal damage, because the illusion that they are in the correct way made them to be sure that there is no chance that their theories can be wrong. Two neutrons do not bind via strong force by the simple reason that strong force does not exist. The interaction between two protons, detected in the proton-proton scattering experiments, interpreted by theorists as being a strong force, actually must be a kind of dynamic interaction through gravitons, when protons move with relativistic speeds. As consequence, nowadays the situation is tremendously dramatic, because we realize that the rejection of the aether, together with the adoption of strange speculations, have thrown the Theoretical Physics in its worst crisis along the centuries of its development. It's unquestionable that the method of rejecting the aether and adopting strange speculations has failed, and therefore we need to change our way of investigation, otherwise, if we continue using the old failed method, the theorists will never to succeed to find a theory free of paradoxes and puzzles, a theory based on foundations that reflect those foundations existing in the nature and rule the laws responsible for the physical 
phenomena. Thereby, we conclude that aether must be reintroduced, and reasonable speculations must be tried in the process of discovery of the fundamental principles that rule the working of the universe. This is the method tried in the Guglinski-Nassif theory.

\section{PROTON RADIUS SHRINKAGE}

The measurement of the neutrino speed, with the ICARUS detector (Antonello et al., 2012), plays for the advancement of the Theoretical Physics, in 2012, the same role played by the Michelson-Morley experiment, in 1887. Unfortunately, in general the generation of scientists, contemporary to revolutionary breakthrough experiments, do not realize that they are witnessing the birth of New Physics, where some fundamental principles, which they believed to be definitive and untouchable, will be changed, or replaced by new ones. In 2010 an international research team published a proton charge radius measurement via the Lamb shift in muonic hydrogen. Their measurement of the root-mean-square charge radius of a proton is $0.84184(67) \mathrm{fm}$, which differs by 5.0 standard deviations from the CODATA value of 0.8768(69)fm (Randolf, Aldo, \& François et al. 2010). In January 2013, an updated value for the charge radius of a proton, $0.84087(39) \mathrm{fm}$, was published.

The international research team that obtained this result is now attempting to explain the discrepancy, and re-examining the results. If no errors are found in the measurements or calculations, it could be necessary to re-examine the world's most precise and best-tested fundamental theory: quantum electrodynamics (QED)(Pohl et al., 2010).

The re-examination of QED is also required as consequence of the measurement of the neutrino speed with the ICARUS detector, because if the neutrinos really move with the speed of light, this imply in the Lorentz factor violation, and, if the mass of elementary particles is, indeed, promoted by the interaction of their electric charges with gravitons filling the space, as proposed in (Cruz, 2016), then something is missing in QED, since the interaction between electric fields and gravitons cannot be explained via QED. Here we propose that there is connection between the two QED violations, one by proton radius shrinkage, and other by Lorentz factor violation.

\section{WHAT IS MISSING IN THE EQUATIONS OF PHYSICS?}

The physicists know that something is missing in the Standard Model (SM). The question is discovering what is missing. In the book "Time Reborn", by Lee Smolin, he writes: "It remains a great temptation to take a law or principle we can successfully apply to all the world's subsystems and apply it to the universe as a whole. To do so is to commit a fallacy I will call the cosmological fallacy". In his opinion, the mathematisation of physics and the reduction of the universe, to a mathematical object, has confused the physicists and accounts for the worst and most distracting pronouncements of physicists. The concept on which he bases his thesis is Time. He thinks that, as time has been excluded from physics, the equations of physics have the property of being timeless and describe a world where Time plays no any role, whereas, unlike, this does not occur in the natural world. Of course many conjectures can be supposed on what is missing in the equations of physics. However, first of all, we have to remember that gravity is missing in the SM. Then, instead of considering the Time, or other any cause, as the villain responsible for the crisis in the Theoretical Physics, it seems obvious that gravity deserves to receive the title of villain much more than Time, or other causes. Besides, as the aether is missing in the current theories of Modern Physics, and, in the case the aether really exists filling the space, and gravitons are particles of the "soup" composed by particles of the aether, then it seems to be obvious that gravity is really the villain responsible for the crisis in Physics. And if the theorists finally recognize that what is missing in the equations of the current theories of Physics is related to the missing of aether, then many theories will be proposed by several authors, in which the equations will receive the introduction of the aether contribution.

In the field of the macroscopic world, a theory which is being successful for the obtainment of several results agree to experimental findings is the Symmetric Special RelativitySSR (Cruz, 2016; Cláudio Nassif, 2008; Claudio Nassif, 2010; Cláudio Nassif, 2012, 2015), where, together with the Einstein's upper limit of speed for macroscopic bodies, is proposed a lower limit of speed for elementary particles, introducing by this way a symmetry for the Theory of Relativity.

For the macroscopic world, the task of discovering new equations, where is introduced the participation of the aether, is relatively very easier than in the case of microscopic world, because for the macro world it is possible to start from the old well-known equations, in which the contribution of the aether is missing, and the task is summarized in the search of what is missing. In the field of microscopic world, covered by atomic, nuclear, and particle physics, the task is harder, because the same method applied for the macroscopic world cannot be applied, and the reason is because, first of all, there is need to discover the physical micro-structures of the elementary particles, and the structures of the atom and the atomic nucleus. Of course, in such an enterprise, it's mandatory discovering one unique model for the nucleus, which must be able to explain all the nuclear properties of nuclei (in a different way of that adopted in the current Nuclear Physics, where several nuclear models were adopted, and each one of them is applied in order to explain a certain specific narrow range of nuclear properties). The discovery of a unique model is, obviously, a philosophical requirement, based on the assumption that nature cannot produce the nuclear phenomena by working with several different nuclear structures. But the theorists need prevent that their aversion to a philosophical criterion will move them away from the pursuit of truth, because if a sole nuclear model is not found, the theorists may be sure that they are going in the wrong way. Nature does not work with equations. She works with physical structures. Along decades physicists were on the trail of equations, but from now the task should be to find out how existing structures in nature generate the equations.

\section{ON THE CRITERION OF CONFIRMATIONS OF A THEORY BY EXPERIMENTS}

A theory developed through equations is able to make predictions, which may be confirmed, or denied, by experiments. However, even if such theory is confirmed by an experiment, we cannot be sure, without any doubt, that it is correct, because it can be denied by other posterior more 
accurate experiments. Sometimes it seems a prediction of a theory was confirmed, but later the physicists realize that it was only a coincidence, as occurred with prediction of the meson by Yukawa. Nowadays the physicists know that his model of neutron is wrong. On another hand, the physicists rejected the Bohr model of hydrogen atom, which was unable to explain the fine structures, albeit his theory has obtained spectacular results, as for instance the calculation of the Rydberg's constant, with an accuracy impossible to be accidental. And it was already shown that, although Bohr hydrogen atom model is wrong, however the predictions of his theory are not accidental, because there is a centripetal force on the electron, due to the electron trajectory with zitterbewegung motion (Guglinski, 2018b). As is "NO" the answer for the question "can the electron be submitted to a centripetal force in the hydrogen atom of Quantum Mechanics?", then by consequence the atom model of Quantum Mechanics cannot be hundred percent correct. By starting from a theory developed through a physical model, one becomes able to make predictions if he discovers the equations governing the working of the model, as he also may verify if the model is able to supply results agree to experimental data. Along the development of the Quantum Mechanics, the theorists had supposed to be impossible to develop a theory based on physical models, because no chance or uncertainty is connected with the predictions of, say, the laws of motion. So, Quantum Mechanics was developed from a wrong belief, because the introduction of the zbw of the elementary particles reintroduces the uncertainty in the laws of motion. A theory developed through physical models has an important virtue, because if the model disagrees to experimental data, we can be hundred percent sure that the theory is wrong. For instance, suppose that one proposes a nuclear model, according to which even-even nuclei, with equal number of protons and neutrons, $Z=N$, have spherical shape. In the case the experiments detect that those nuclei have ellipsoidal shape, then we can be sure, without any doubt, that his model is wrong, and therefore the foundations of the theory are wrong. When we test a theory, we have to make distinction between two sort of experimentsas,

-Quantitative experiments. They compare the data supplied by the theory with the data supplied by experiments.

-Qualitative experiments. They do not supply numbers, they give only the following two answers: YES or NO.

Michelson-Morley was a qualitative experiment. When Michelson and Morley did it, they did to nature the question “does luminiferous-aether exists?". And their experiment has transmitted to them the following negativeanswer of the nature, "No". However, what the experiment had rejected was the existence of the luminiferous-aether, meaning that light is not a wave moving in a medium, as believed the physicists in the 19th Century. The experiment did not reject the existence of the aether itself. Therefore, it is important to pay attention to what question we ask to nature, when we perform experiments. Other example of qualitative experiment was the detection of the pear shape of the nucleus 88Ra224 (Gaffney et al., 2013). According to the Standard Nuclear Physics (SNP), an even-even nucleus cannot have a pear shape, and therefore the conclusion is clear: the foundations of the SNP cannot be correct. Regarding the Higgs boson, it seems its prediction is similar to the Yukawa's wrong prediction of the meson, with a difference: the mass of the meson was calculated, but Higgs theory did not predict the boson mass.
Intriguingly, the detection of neutrinos moving with the speed of light, required the rejection of the hypothesis that Higgs boson is responsible for the mass of elementary particles, and therefore, one had to expect that the velocity of neutrinos should be measured with ICARUS until exhaustion, in order to bring a definitive answer for the question either neutrinos move with the speed of light, or not. A definitive positive answer "YES" imply that Higgs theory is wrong, and the boson detected in 2012 is not the cause which promotes mass to particles, as proposed by Higgs. As seen, the confirmation or rejection of a theory (or a theoretical model), by experiments, is a complex process, because sometimes it involves even the proud of the physicists, when they anxiously expect hearing the answer "yes" from nature. And the partial confirmation of a theory, as for instance of the Bohr theory of the hydrogen atom, imply in the partial rejection of Quantum Mechanics, because something is missing in the atom model of the theory. On other hand, the detection of a boson in the LHC experiment in 2012, which the physicists had interpreted as being the boson predicted by Higgs, was placed under suspicion (by the experiment which detected neutrinos moving with the speed of light, also in (Antonello et al., 2012).

\section{WHAT IS MISSING IN THE EQUATIONS OF NUCLEAR PHYSICS?}

Regarding the omission of the contribution of the aether in equations, among the branches of Theoretical Physics, one in which the omission has promoted the worst damage is the nuclear physics, and the reason is because the structure of the nucleus is more complex than the nuclear theorists have supposed. Along more than a century, the physicists tried to discover the structure of the atomic nucleus by believing that the endeavor could be successful by simply applying, to the nucleus, the laws of Quantum Mechanics which were successfully applied to the atom. The enterprise has failed, as we realize from several experiments, published along the last decade, which have invalidated all the current nuclear models, conceived under the principles of the SNP. This suggests that the structure of the nucleus must be discovered by discovering, firstly, its physical structure, where the contribution of the particles of the aether plays a fundamental role.

In 1993 the author of the present paper had undertaken a deep investigation in the field of Nuclear Physics. And he has arrived to the conclusion that any current nuclear model, (where a nucleon moves-having interaction with other ones by only Coulomb forces, strong nuclear forces, and spininteractions) is not able to reproduce the nuclear magnetic moment of some nuclei. Then he understood that there was need to consider a model where the nucleons are captured by a sort of strings formed by magnetons. Obviously there was need to consider a source for the production of the strings, and then the author had concluded that all nuclei have a central nucleon $2 \mathrm{He} 4$, responsible for the production of the strings. Later he has realized that even-even nuclei with $Z=N$, formed by nucleons captured by those strings, composed by a flux of magnetons, could not have null magnetic moment, and therefore there was need to suppose that those strings were formed by a flux of other elementary particles of the aether, instead of magnetons. And so the magnetons were replaced by gravitons. Probably most of the physicists reading now about strings inside the nuclei will consider such hypothesis too much speculative. However, what to say about the superstring 
theory? Superstring is an attempt to explain all of the particles and fundamental forces of nature in one theory by modelling them as vibrations of tiny supersymmetric strings. Isn't a speculation? And if one accepts to think about particles and fundamental forces as strings, then why cannot he think about strings inside the nuclei?

Figure 1 shows that the rotation of quarks (in the structure of the proton) induces the strings composed by pure gravitons $g(+)$. Here, "pure" means that only gravitons compose the strings (the strings do not capture electricitons).

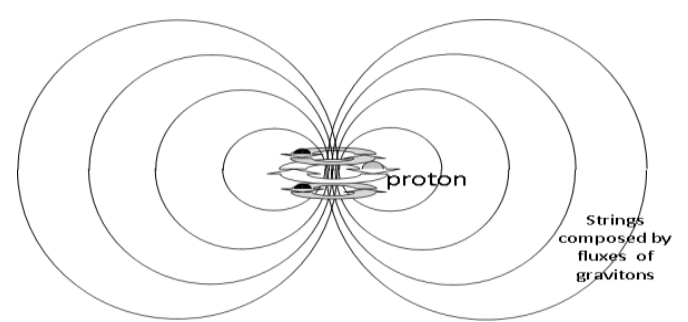

Fig 1. Flux of pure gravitons $g(+)$ induced by rotation of quarks

As said, all the nuclei have a central nucleon $2 \mathrm{He} 4$, whose strings of gravitons captures protons and neutrons. Figure 2 shows the structure of $3 \mathrm{Li7}$, where one proton and one neutron were captured by the strings of gravitons (the neutron does not leave the $L i 7$ because it has spin-interaction with the deuteron).

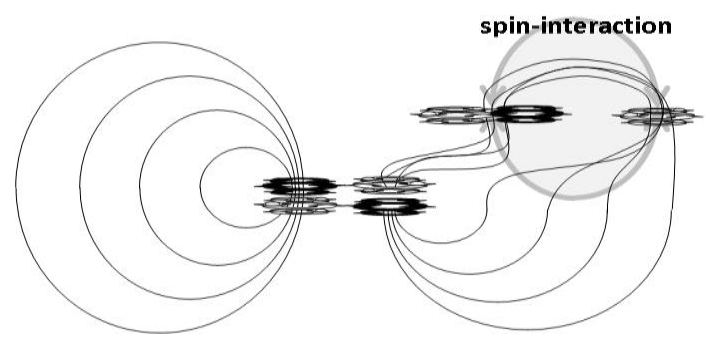

Fig 2. Structure of $3 \mathrm{Li7}$

Several hexagonal floors are formed around the central $2 \mathrm{He} 4$. Figure 3(A) shows the complete hexagonal floor of the nucleus 8016. Due to Coulomb repulsions, the six deuterons oscillate, in order that the structure of 8016 is not flat, but actually it assumes an ellipsoidal shape, as shown in 3(B), where the strings of gravitons are not shown. All the light even-even nuclei with $Z=N$ have ellipsoidal shape, and in Figure 3(C) it is shown the structure of 14 Si28, formed by two complete hexagonal floors. The new nuclear model was baptized as "Hexagonal Floors Model". With the growth of the quantity of hexagonal floors, even-even nuclei approach the spherical shape, as for instance the $92 U$.

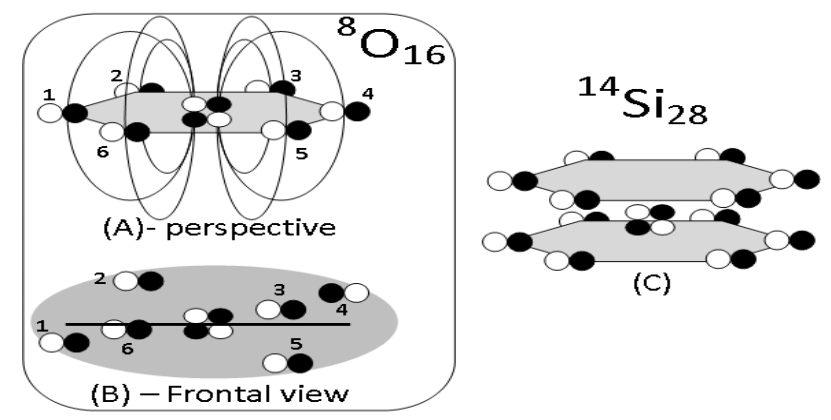

Fig 3. Hexagonal floors of $0_{16}^{8}$ and $14 \operatorname{Si} 28$
According to the SNP, the even-even nuclei with $Z=N$ cannot have ellipsoidal shape, and therefore the nuclear model with hexagonal floors could not be considered seriously by nuclear theorists, because they knew not only that the principles of the SNP requires a spherical shape for those nuclei, but also because they knew those nuclei have null electric quadrupole moment, and therefore it was mandatory they have spherical shape. Besides, as in that new nuclear model there is a central $2 \mathrm{He} 4$, and the nucleons are captured by a string formed by a flux of gravitons (instead of be bound by strong nuclear force, as considered in all current nuclear models), the nuclear theorists had more strong reasons why do not consider seriously a "strange" model formed by hexagonal floors. Obviously the author was aware that a paper, proposing the exotic new nuclear model, would never be accepted for publication in any reputable peer journal of physics. That's why in 2004 he has decided to meet his several papers in a book form, and to look for a publisher. In the end of 2005 an editor has accepted to publish it, and the book was published in August 2006, with the title Quantum Ring Theory (QRT) (Guglinski, 2006). Spherical distribution of charges has null electric quadrupole moment, $Q=0$, while ellipsoidal distribution elongated toward $Z$-axis has $Q>0$, and elongated toward $\mathrm{XY}$ plane has $\mathrm{Q}<0$. As experiments already had detected that even-even nuclei with $Z=N$ have $Q=0$, then obviously the author had to justify how, in spite of they have ellipsoidal shape, however they have $Q=0$. The argument, which justifies why they have $Q=0$, is proposed in the page 137 of the book QRT.

Another prediction was regarding the distribution of the nucleons, because, as they occupy places in the corners of hexagonal floors distributed about the Z-axis, then in the Hexagonal Floors Model there is a preferential direction of distribution. In the page 133 is written. The distribution about the z-axis is a nuclear property up to now unknown in Nuclear Physics. And obviously such prediction, of the existence of a preferential direction for the distribution of the nucleons, along the Z-axis, was other strong reason for rejection of the new nuclear model, because, according the foundations of the SNP, a preferential direction of distribution of nucleons is impossible. In 2012 the journal Nature published a paper demolishing a dogma of current nuclear physics, considered untouchable along 80 years, reporting experiments which detected that even-even nuclei with $Z=N$ have ellipsoidal shape (Ebran, Khan, Nikšić, \& Vretenar, 2012). In 18 July 2012 the nuclear theorist Martin Freer had published in News \& Views, by Nature, an article (Nuclear physics: Nucleons come together), and the author sent him the comment ahead.

Dear Martin Freer, With that distribution of charge of the Ne20 structure shown in Figure 1, how to explain that Ne20 has null electric quadrupole momentum? That structure shown in Figure 1 is not spherical, and therefore Ne20 could not have null electric quadrupole momentum (detected in experiments concerning nuclear data). Martin sent the reply ahead.

The nucleus is intrinsically deformed as shown, but has spin 0 . Consequently, there is no preferred orientation in the laboratory frame and thus the experimental quadrupole is an average over all orientations and hence is zero. Experimentally is possible to show that the deformation of the ground state is non zero by breaking the symmetry and rotating the nucleus. Martin.

However Martin's explanation requires that $\mathrm{Ne} 20$ has rotation in the ground state, because his argument ( there is no preferred 
orientation in the laboratory frame) does not mean that $\mathrm{Q}(\mathrm{Ne} 20)$, detected in experiments, must be zero, because if $\mathrm{Ne} 20$ does not rotate, then the experiments had to detect NON null Q(Ne20).

If $\mathrm{Ne} 20$ had no rotation in the ground state, then $\mathrm{Q}(\mathrm{Ne} 20)$ detected as null in the experiments actually would mean that there is $\mathrm{NO}$ way to know if $\mathrm{Q}(\mathrm{Ne} 20)>0$ or $\mathrm{Q}(\mathrm{Ne} 20)<0$, because "there is no preferred orientation in the laboratory", and so there is NO way to know in what direction Ne20 has oblong shape, regarding any axis. But the experiments have detected $\mathrm{Q}(\mathrm{Ne} 20)=0$ because $\mathrm{Ne} 20$ really rotates in the ground state, and only from the hypothesis of its rotation Martin's argument gets sense (his argument that experimental quadrupole is an average over all orientations and hence is zero).

Therefore, the discovery that $10 \mathrm{Ne} 20$ has ellipsoidal shape requires that even-even nuclei with $\mathrm{Z}=\mathrm{N}$ have rotation at the ground state. But the most interesting is that Martin's argument is basically the same proposed in the page 137 of the book QRT, published in 2006, where it is explained why oxygen-16 has $Q(016)=0$, in spite of it has ellipsoidal shape, as follows. Note that as the $0_{16}^{8}$ has a null nuclear magnetic moment $\mu=0$, then its nuclear spin cannot be aligned toward a direction by applying an external magnetic field, and so its nuclear spin can indeed be chaotic. So the $x-y$ plane has a chaotic rotation, and the six nucleons $1 \mathrm{H} 2$ performs the surface of a sphere, and the $\mathrm{z}$-axis has a chaotic rotation around the center of the nucleus $0_{16}^{8}$. By consequence the $0_{16}^{8}$ behaves like if it should be a spherical distribution of positives charges, and not a flat distribution. That's why $\mathrm{O}_{16}^{8}$ has $\mathrm{Q}(016)=0$.

Therefore, six years before the author had proposed the same argument used by Martin Freer. But there is another puzzle, and it is impossible to be solved, because the authors of the paper published by Nature have shown "how" the atomic nuclei cluster, however they do not show "why" they cluster in that way, and they did not explain it because, by considering the principles of the SNP, protons and neutrons cannot take places in order to form an ellipsoidal distribution inside the even-even nuclei with $Z=N$. In another words, the "physical" cause (which obliges the nucleons of taking their positions, in order to form a structure with ellipsoidal shape), cannot be found by considering the foundations of the SNP. That would require the existence of a fifth force in nature, or other speculation. Other interesting question is about the argument proposed by Martin Freer, because his proposal makes sense only if we consider that even-even nuclei with $Z=N$ have rotation in the ground state. But this is impossible by considering the SNP, because the rotation of the charge of protons in the same direction would induce a magnetic moment, whereas experiments have detected null magnetic moment for those nuclei. Such puzzle can be solved only by considering a new nuclear model by considering a structure for the aether, according to which (in the case of a rotation of the nucleus as a whole) proton rotation does not induce magnetic moment, when it rotates in the ground state. As seen, the SNP is in very trouble situation. Because even if the nuclear theorists decide to save it by proposing a fifth force, such solution is unable to explain why even-even nuclei with $Z=N$ have null magnetic moment, in spite of they rotate in the ground state. The prediction that nucleons have a preferential distribution along the Z-axis (as proposed in the page 133 of the book QRT) was confirmed in 2013, by an experiment which detected that $88 R a 224$ has pear shape (Gaffney et al.,
2013). But according to the SNP, the nucleus Ra224 cannot have pear shape, because from SNP foundations all the eveneven nuclei must have either a spherical shape (when $Z=N$ or an ellipsoidal shape (when $N>Z$ ). In the homepage of Science \& Technology it's written that Professor Peter Butler from the University of Liverpool, (the nuclear physicists who led the research) commented that they have been able to show that while Radium 224 is pear-shaped, Radon 220 does not assume the fixed shape of a pear but rather vibrates about this shape, and from his opinion the details of those findings are in contradiction with some nuclear theories and will help others to be refined. The experimental observation of nuclear pear shapes is not only important for understanding the theory of nuclear structure and how nuclei behave, but also in answering the broader questions about the fundamental interactions that govern the structure of the universe. In May 10, 2013, the homepage Science \& Technology News has published the article Physicists Discover First Direct Evidence of Pear Shaped Nuclei in Exotic Atoms, where is written that physicists have been searching for signs of a new force or interaction that might explain the matter-antimatter discrepancy, and the evidence of its existence would be revealed by measuring how the axis of nuclei of the radioactive elements radon and radium line up with the spin. And Dr. Timothy Chupp, a University of Michigan professor of physics, has explained how the theorists are dealing with the puzzle. His opinion is that pear shape is special. It means the neutrons and protons, which compose the nucleus, are in slightly different places along an internal axis. The new interaction, whose effects we are studying does two things. It produces the matter/antimatter asymmetry in the early universe and it aligns the direction of the spin and the charge axis in these pear-shaped nuclei.

In resume, in 2013 the discovery that Ra224 is pear shaped confirmed what was predicted in the page 133 of the book QRT, published in 2006, where the preferential distribution of protons and neutrons in atomic nuclei was proposed as; The distribution about the $\mathrm{z}$-axis is a nuclear property up to now unknown in Nuclear Physics (Guglinski, 2006).

\section{PRINCIPAL AND SECONDARY FIELDS OF THE PROTON}

Figure 4 shows the principal field $\mathrm{Sp}(\mathrm{p})$ of the proton, composed by two fields formed by strings of gravitons, a central field surrounded by the second field.

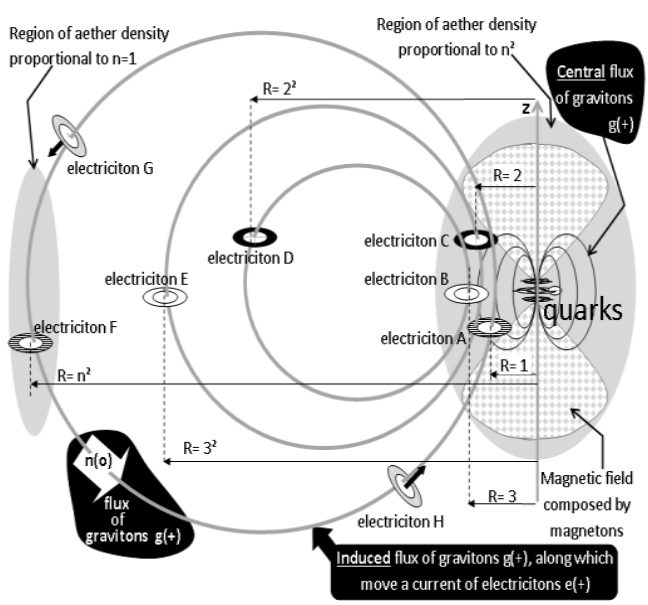

Fig 4. Principal $S p(p)$ field of the proton (shown only the left side). 
In Figure 5 the $\mathrm{Sp}(\mathrm{p})$ field is composed by two fields as -the central n(o)-flux of gravitons $g(+)$

-and the induced n(o)-flux of gravitons $g(+)$, along which electricitons $e(+)$ move with the speed of light

The rotation of the three quarks of the proton induce the central field, composed by fluxes of pure gravitons $g(+)$. The radius of this central field has an extension in the order of few femtometers. The rotation of the fluxes of this central field induces the second field, which radius has an extension in the order of Bohr's radius, and is composed by strings of gravitons $g(+)$, where each string captures a lot of positive electricitons $e(+)$, which move along the strings with the speed of light. Such strings of gravitons is named $\mathrm{n}(\mathrm{o})$-flux. In the $\mathrm{Sp}(\mathrm{e})$ principal field of the electron, the $\mathrm{n}(\mathrm{o})$-flux is composed by strings of gravitons $g(-)$, where each string captures a lot of negative electricitons $e(-)$.

The electromagnetic fields of the photon are also composed by electricitons (Guglinski, 2018b).

Note: Signs within parentheses of gravitons $g(+)$ and $g(-)$ does not means that they have positive and negative charges. It only means that $g(+)$ induces a flux of $e(+)$, while $g(-)$ induces a flux of $e(-)$.

The external principal field has rotation, but, albeit it has an extension of the Bohr's radius and it is composed also by electricitons, its rotation does not induce magnetic moment, because it is immersed in an anisotropic aether, and the reason why its magnetic moment is null we realize from the Figure 4, as explained ahead.

1. Electriciton A is situated in a region where the density of the aether is proportional to $n^{2}$, whereas electriciton $\mathrm{F}$ is situated in a region where the density is proportional to $n=1$. While the quantity of magnetons in the place occupied by the electriciton $\mathrm{A}$ is proportional to $n^{2}$, the quantity of magnetons in the place occupied by $\mathrm{F}$ is proportional to 1 .

2. Electricitons A and $\mathrm{F}$ have contrary spins

3. Electriciton A rotates with radius $R=1$, whereas electriciton $\mathrm{F}$ rotates with radius $R=n^{2}$.

4. The magnitude of a magnetic field is proportional to the density of the aether where the field is induced. So, electriciton $\mathrm{A}$ and $\mathrm{F}$ induce two magnetic fields which cancel one each other.

The principal field $\mathrm{Sp}(\mathrm{p})$ of the proton rotates chaotically together with the chaotic rotation of its body composed by quarks, and the rotation of $\mathrm{Sp}(\mathrm{p})$ induces a secondary field, named $\mathrm{Sn}(\mathrm{p})$, formed by rectilinear strings composed by electricitons $e(+)$, which move with the speed of light.

The structure of the secondary field is shown in detail in (Guglinski, 2018b). Figure 5 shows the principal and secondary fields of the proton. As explained in (Guglinski, 2018 b), whereas $S p(p)$ rotates, the secondary field $S n(p)$ does not rotate, because it does not belong to the proton. The secondary field $\mathrm{Sn}(\mathrm{p})$ actually belong to the rest of the universe, but when the proton moves it drags together the secondary field. The secondary field promotes the electric properties of the proton. In the neutron the secondary field $\mathrm{Sn}(\mathrm{n})$ is neutral, because it is the overlap between $\mathrm{Sn}(\mathrm{p})$ and $\mathrm{Sn}(\mathrm{e})$, which are respectively the secondary field of the proton and electron. Beyond the secondary electric field $\mathrm{Sn}(\mathrm{p})$, the rotation of the $\mathrm{Sp}(\mathrm{p})$ principal field of the proton also induces a field formed by rectilinear strings of gravitons, responsible for the gravitational interactions of the proton. This field also belongs to the rest of the universe. The secondary electric field and gravitational field are concentric, and their strings spread together along the universe. So we had to expect that electric and gravitational field had the same range of action. But it is known experimentally that electric fields actuate in short distances, and gravitational fields is far-reaching. The reason why electric fields are short-range is shown in the Figure 2 in (Guglinski, 2018b).

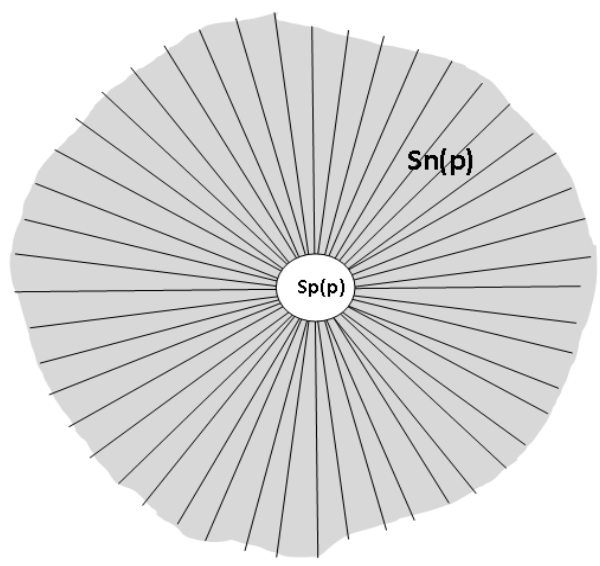

Fig 5. Rotation of principal $S p(p)$ field induces the secondary $S n(p)$ field. The $\operatorname{Sn}(\mathrm{p})$ field is responsible for the proton electric charge $+1 e$

According to (Guglinski, 2018b), the general Coulomb law is $F=K\left(Q q / d^{1 / X}\right)$, and the relations between " $\mathrm{x}$ " and " $\mathrm{d}$ " are the follows

1) $\mathrm{x}=0.5$ when, $\mathrm{d}>10^{-11} \mathrm{~m}$ (Bohr radius)

2) $0.5<\mathrm{x}<1.0$ when, $10^{-14} \mathrm{~m}<\mathrm{d}<10^{-11} \mathrm{~m}$

3) $\mathrm{x}>1.0$ when, $\mathrm{d}<10^{-15} \mathrm{~m}$, and $X$ increases as $d$ decreases.

This is one of the fundamental things missing in the equations of the SNP.

\section{SHRINKAGE OF THE PROTON RADIUS}

The electric fields of the quarks interact with a Coulomb force $\mathrm{F}=\mathrm{K} . \mathrm{Q} \cdot \mathrm{q} / \mathrm{d}^{1 / \mathrm{X}}$, as occurs with the proton and the electron, and in general with any particle with electric charge, when $d<$ $10^{-15} \mathrm{~m}$. Then the repulsion Coulomb force between the two up quarks of the proton, separated by a distance shorter than $2 \mathrm{fm}$, is not so strong as the nuclear theorists believed. The repulsion is relatively weak, and the quarks are bound via the partnership between the gluons and the flux of gravitons induced by the rotation of the quarks.

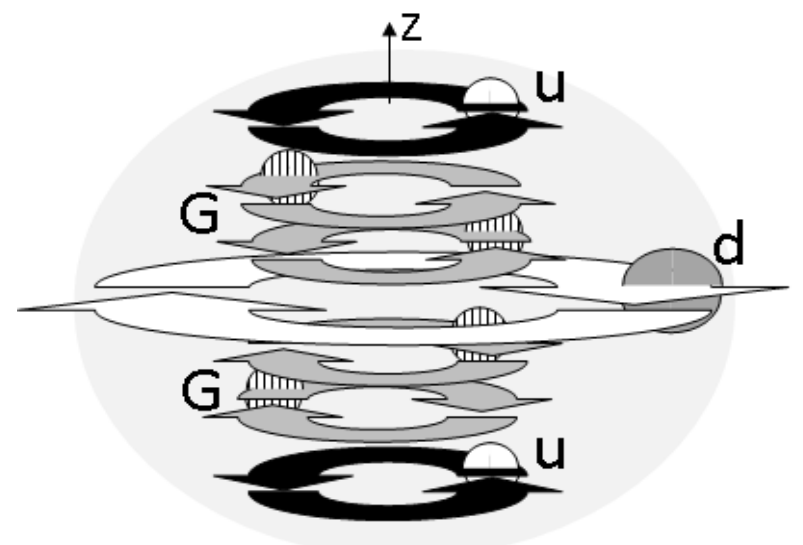

Fig 6. Proton formed by two up quarks, one down quark, and two big G gluons (Guglinski, 2018c). 
The structure of the proton body is shown in Figure 6. As the three quarks have a very fast rotation, they are submitted to a strong inertial force (centrifugal), which tries to break down the proton body. The interaction between the $n(0)$-flux of gravitons and the electric charge of the quarks counteracts the centrifugal force, promoting the balance of the quarks in the radial direction. As the down quark has charge $-1 / 3$, alone it cannot counteract the repulsion between the charges $+2 / 3$ of each of the up quarks. So the weak Coulomb repulsion between the up quarks along the $\mathrm{z}$-axis is counteracted thanks to the help of the gluons, and also the magnetic interactions between the down quark and the two up quarks.

As the counterbalance of the centrifugal force on the quarks is promoted by the $\mathrm{n}(\mathrm{o})$-flux of gravitons induced by the rotation of the quarks, the proton radius depends on the intensity of the $\mathrm{n}(\mathrm{o})$-flux of gravitons. When the proton interacts with a neutron, the gravity $\mathrm{n}(\mathrm{o})$-flux of the proton is reinforced by the $\mathrm{n}(\mathrm{o})$-flux of the neutron, and so the proton radius has a shrinkage. As the radius becomes shorter, the quarks start to move faster, so that to increase the centrifugal force, $F_{C}=$ $m \omega^{2} R$, (because it decreased due to the shrinkage of the radius). As the quarks move faster, the intensity of the gravity $\mathrm{n}(\mathrm{o})$-flux grows, and so it is established the equilibrium of forces in the radial direction, thanks to the shrinkage of the proton radius.

Therefore, there is no need to consider the asymptotic freedom for justifying why quarks interact strongly at low energies, preventing the unbinding of baryons. And the reason why there are no free quarks is because the n(o)-flux, of each free quark, is not able to counterbalance the centrifugal force on it, but the $\mathrm{n}(\mathrm{o})$-flux due to three quarks of a proton is able to promote the equilibrium between their interaction force with the n(o)-flux and the centrifugal force. The stability of a hadron formed by three quarks depends on their masses. Quarks as charm (c), strange (s), top (t), and bottom (b), cannot form a stable hadron, because the rotation of their charges (combinations of $+2 / 3$ and $-1 / 3$ ) induce a gravity $\mathrm{n}(\mathrm{o})$-flux in the same magnitude of that induced by up and down quarks, and as the masses of $c, s, t$, and b, are very larger (than the masses of up and down quarks), then the gravity n(o)-flux cannot avoid that they be expelled by centrifugal force. As we realize, the shrinkage of the proton radius is consequence of the interactions of electric charges (of the quarks with rotation) and the n(o)-flux. In another paper published by Guglinski, to published later, it is shown that the growth of the mass of particles (by following the Lorentz factor, as proposed by Einstein) is promoted by the interaction between electric charges of elementary particles and the gravitons existing in the space filled by the aether, corroborating what is proposed in (Cruz, 2016), but Guglinski's paper introduces an additional argument, because Nassif's theory is unable to explain why neutral pions and the neutron have mass, in spite of they have no charge. Therefore, the shrinkage of the proton radius and the growth of mass by Lorentz factor, are both under the rule of the law of interaction between electricitons and gravitons.

\section{THE STRUCTURE OF THE LEPTONS AND THE ORIGIN OF THE WEAK NUCLEAR FORCE}

As proposed in a paper to be published, the structure of the photon is formed by two singletons $S(+)$ and $S(-)$, both them composed of several electricitons. The structure of the photon is $S(+) \cdot g . g . S(-)$, where $S(+)$ and $S(-)$ have contrary spins, and the two gluons " $g$ " with spin $1 / 2$ have parallel spins.

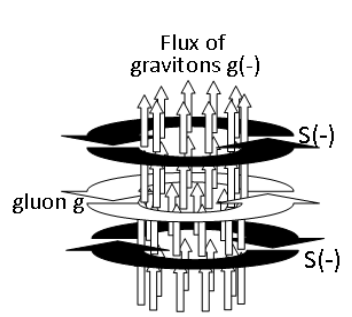

(A) : electron

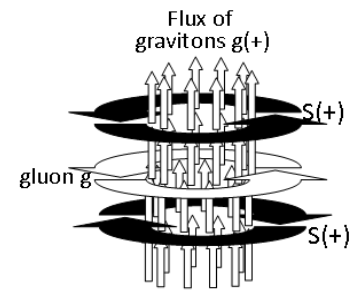

(B) : positron

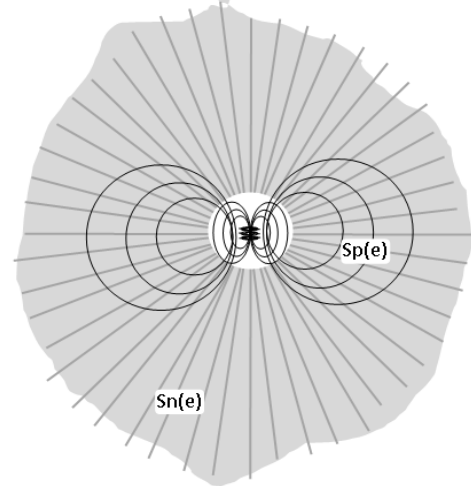

(C) : Electron with its fields Sp(e) and Sn(e)

Fig 7. Differences in the structures of the electron and positron Consider the electron-positron annihilation with the production of two gamma photons:

$$
e^{+}+e^{-} \rightarrow \gamma+\gamma
$$

which we can put as ahead

$$
e^{+}+e^{-} \rightarrow S(-), g, g, S(+)+S(+), g, g, S(-)
$$

We have good reasons for supposing that leptons are composed by a sandwich of one gluon with spin $1 / 2$ and two singletons with contrary spins. So, the structure of the electron is $S(-), g, S(-)$, where each singleton $S(-)$ has charge $-0.5 e$, and the positron structure is $S(+), g, S(+)$. Thereby the electron-positron annihilation shown in Eq (2) is,

$$
\begin{gathered}
S(-), g, S(-)+S(+), g, S(+) S(-), g, g, S(+) \\
+S(+), g, g, S(-)
\end{gathered}
$$

The cause of the stability of leptons with no charge, as the neutrinos, is shown in a paper to be published. And the stability of the charged leptons depends on the equilibrium in the dispute established by the centrifugal force against the force generated by the interaction between the singletons charge and the n(o)-flux. Only the electron and the positron are stable, because they are composed by singletons with the suitable mass, in order to be established the equilibrium between the centrifugal force and the interaction force between singletons and the n(o)-flux. Figures 7(A) and 7(B) show the differences in the structures of the electron and positron. In Figure $7(\mathrm{C})$ is shown the two fields of the electron, the principal and the secondary. 
Concerning the question on why does not exist antimatter in the universe, the answer must be found in some asymmetry of the aether structure. For instance, the following asymmetry. 1 - There is a permeability particle " $\mathrm{P}$ " which promotes the interaction between gravitons $g(+)$ and electricitons $e(+)$.

2 - There is a permeability particle " $p$ " which promotes the interaction between gravitons $g(+)$ and electricitons $e(-)$.

3 - As the singletons $S(+)$ of the positron are crossed by a n(o)flux of gravitons $g(+)$, then when occurred the Big Bang the positrons were not created, because the interaction between gravitons $g(+)$ with particles $\mathrm{P}$ was suitable of producing only protons, and the interaction between gravitons $g(-)$ and particles $\mathrm{p}$ was suitable of producing only electrons.

4- Therefore, antiprotons were not produced, because their quarks are crossed by a n(o)-flux of gravitons $g(-)$, and their interaction with the particle $\mathrm{p}$ is not suitable to produce antiprotons, while the positrons were not produced because their singletons are crossed by a n(o)-flux of gravitons $g(+)$, and their interaction with the particle $\mathrm{P}$ is not suitable to produce positrons.

The evidence, in the form of extra electrons and positrons emitted at a particular angle, in the anomalous internal pair creation in Be8 (Krasznahorkay et al., 2016) is probably consequence of the asymmetry in the structure of the aether, no matter if due to asymmetry caused by the particles $\mathrm{P}$ and $\mathrm{p}$, or if caused by asymmetry of other two particles of the aether. As we realize from the structure of the leptons, their principal field $\mathrm{Sp}(\mathrm{L})$ promotes weak electromagnetic interactions, because as $\mathrm{Sp}(\mathrm{L})$ is induced by the rotation of two singletons, the strings of $\mathrm{Sp}(\mathrm{L})$ composed by flux of gravitons is weaker if compared with the strings of $\mathrm{Sp}(\mathrm{H})$ of hadrons, composed by flux of gravitons induced by the rotation of three quarks (up quark is a singleton with charge $\mp 2 / 3$ and down quark is a singleton with charge $-1 / 3$ ).

The reason why the singletons of leptons have charge $0.5 e$ and the singletons of hadrons have charge $2 e / 3$ and $1 e / 3$ is related to the fact that the singletons of leptons are bound by gluons with spin $1 / 2$, whereas the singletons of hadrons are bound by the big gluons $\mathrm{G}$ with spin 1 . So we have to conclude that there are two sort of gluons, the hadronic and the leptonic. And the leptonic gluons are divided in electronic, muonic, and tauonic, and probably the three sort of leptonic gluons share the property to morph from one flavor to another, as occurs in the case of the neutrinos. Probably this ability of the neutrinos is also connected with the asymmetry of the aether caused by the particles $\mathrm{P}$ and $\mathrm{p}$, by considering that gluons in some way interact with the particles $\mathrm{P}$ and $\mathrm{p}$.

\section{GRAVITATIONAL ORIGIN OF THE STRONG NUCLEAR FORCE}

Experiments have shown that, in proton-neutron scattering, when they interact with contrary spins, the interaction force is $40 \%$ weaker that that when they interact with parallel spins. Figure 8 shows why the spin influences the force of interaction proton-neutron. First of all, it is known that there are no deuterons with spin zero in nature. In Figure 8(A) are shown a proton and a neutron separated by a long distance. In $8(\mathrm{~B})$ it is shown the deuteron, and we realize that, in the region of interaction between the fluxes of gravitons (of the proton and neutron), their strings which compose the fluxes move in contrary direction. This promotes a strong "friction" (of course not in the sense of the ordinary friction) between the strings, and in this way proton and neutron interact through the so called strong nuclear force, which actually has gravitational origin.

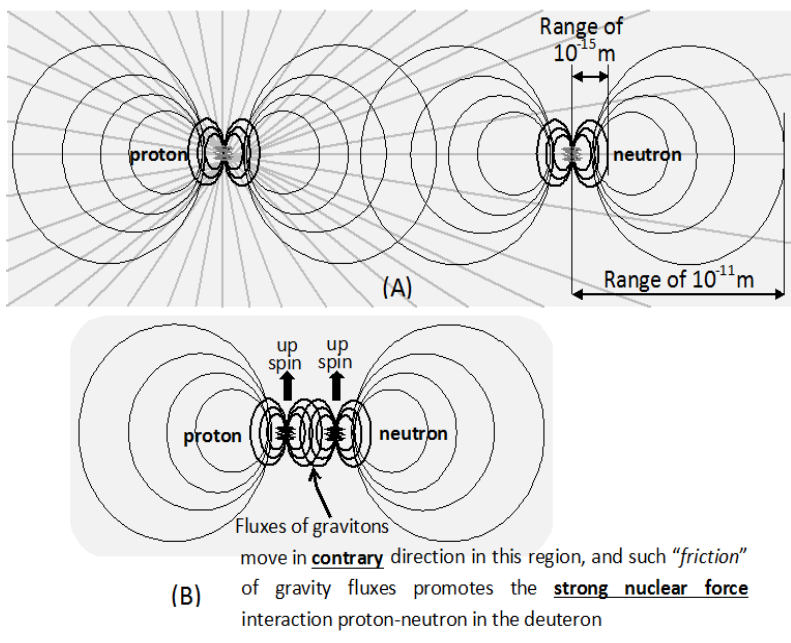

Fig 8. The interaction of gravity fluxes promoting the strong nuclear force between proton and neutron in the deuteron.

Figure 9 shows the structure of the free neutron. As the two up quarks have contrary spins, they cancel each other their tendency of inducing the $\mathrm{n}(\mathrm{o})$-flux. Therefore, the induction of the $\mathrm{n}(\mathrm{o})$-flux is produced almost by the down quark only. The electron contribution obviously is very weak, since it interacts via weak nuclear force.

Now consider the interaction of the central fluxes of gravitons of the proton and electron, when the proton starts to capture the electron, in order to compose a neutron. The cross-section of the interaction is small, and so is weak the interaction proton-electron, because they interact via weak nuclear force. However, when the electron is captured by the proton (and the electron occupies its place inside the newborn neutron), the two fluxes of gravitons (of the proton and electron) overlap hundred percent, and so the total area of interaction is very large. Look at the structure of the neutron in Figure 9. The up quark number 1 and the electron have parallel spins, and therefore they do not interact via weak force. The down quark and the electron also have parallel spins, and they also do not interact via weak force. But the up quark number 2 and the electron have contrary spins, and therefore they have a maximum interaction via weak force (an interaction larger than that obtained via proton-electron scattering experiments, because in those experiments the cross-section is small, whereas inside the free neutron the area of interaction is large). Such mechanism explains the interaction force (via weak nuclear interaction) responsible for the permanence of the electron inside the free neutron, at least along 15 minutes, before decay.

Concerning a neutron bound to a proton (when they compose a deuteron), by looking at the Figure 8(B) one realizes that, beyond the full interaction between the electron and the down quark inside the neutron, the electron also interacts with the down quark of the proton, because looking at the Figure 9, we see that the electron and the down quark of a proton have contrary spins (the proton at the right side of the Figure 9). This additional interaction can be an additional reason why the neutron is stable when bound to a proton, and when inside a nucleus. 


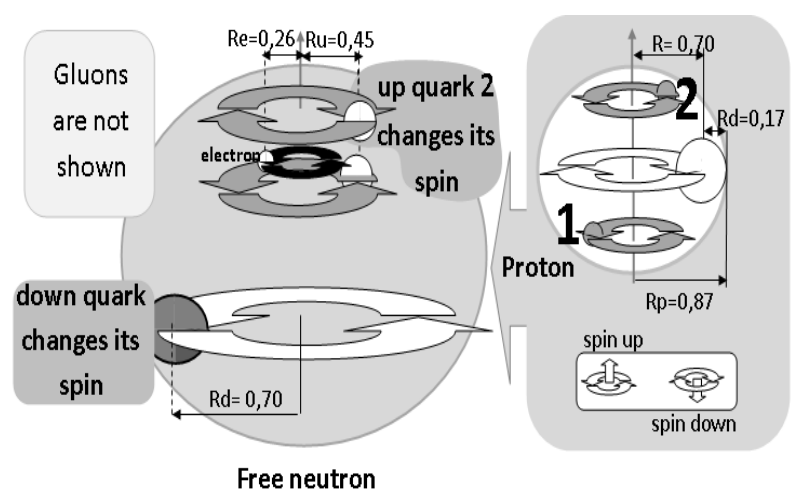

Fig 9. Free neutron structure shown in the paper "Reevaluation of Fermi's theory of beta-decay” (Guglinski, 2018a).

On May 13, 2014, the Scientific American homepage published an article entitled "Neutron Death Mystery Has Physicists Stymied", concerning the difference of $9 \mathrm{~s}$ in the measurement of the neutron lifetime, by two different methods. The article is ended with a prophecy. If neutrons persist in their befuddling behavior, it just might mean the universe is a bit more complicated than we thought. The enigma is not solved yet from the foundations of the SNP, and theorists are so desperate that some of them are proposing nonsenses, as to suppose that a dark matter particle is created when the neutron decays, a speculation already denied by an experiment (Tang, 2018). The answer for the puzzle is proposed in (Guglinski, 2018a), according to which the difference between the two lifetimes of the neutron is related to its shrinkage inside the atomic nuclei (source of neutron for beam experiments), whereas the shrinkage does not occur in the bottle experiments.

\section{FUNDAMENTAL PILARS OF THE STANDARD NUCLEAR PHYSICS DEMOLISHED BY NEW EXPERIMENTAL FINDINGS}

Along the last decade, the advancement of technology has allowed the performance of several experiments measuring the nuclear properties of light atomic nuclei. They are made with the aim of verifying if their nuclear properties fit to what is expected from the principles of the SNP. Surprisingly, the nuclear theorists are realizing that light nuclei have properties that cannot be explained by the current nuclear models, developed from the principles of the SNP. For instance, a research group has, for the first time, in 2012, managed to measure the size of the charge distribution in the atomic nucleus of the highly exotic beryllium-12 isotope. The researchers were surprised to find that the so-called charge radius increases in comparison with that of the beryllium-11 isotope, while the radius of the matter distribution was significantly smaller. These findings contradict the famous shell-model in nuclear physics regarding the structure of atomic nuclei as it was expected that the nuclear charge radius would also be smaller (Krieger et al., 2012). But among other intriguing discoveries, the most astonishing is that nucleons cannot be bound inside nuclei via strong nuclear force, because its range of maximum actuation is less than $3 \mathrm{fm}$, and an experiment has detected that neutron halo in $B e 11$ is separated from the rest of the nucleus by a distance of $7 \mathrm{fm}$ (Nörtershäuser et al., 2009). And any theory, supported by the principles of the SNP, proposed to explain the enigma, is unacceptable and unfruitful, because

a- Suppose a theory is proposed (no matter if based on the principles of the SNP, or even if based on new arguments beyond the SM), however by keeping the belief that nucleons are bound via strong nuclear force.

b- However the theory is unacceptable, because in $97 \%$ of decays $4 B e 11$ transmutes to $5 B 11$, and therefore the neutron does not leave the nucleus.

c- In the $4 B e 11$, the neutron decays into a proton and electron, and the proton turns back to the core (the newborn 5B11). If the strong nuclear force was responsible for the cohesion of nuclei, the proton could never go back to the core, because in a distance of $7 \mathrm{fm}$ it cannot interact with the core via strong force, and the classical Coulomb repulsion between the core and the proton would be so strong that the proton would be expelled from the newborn $5 B 11$.

d- Therefore $5 B 11$ could never be formed in $97 \%$ of the $4 B e 11$ decay.

e- Conclusion: $B e 11$ halo neutron demolishes the fundamental pillar of the SNP.

\section{INTERACTION OF NUCLEONS INSIDE ATOMIC NUCLEI}

In 2013, the Andrea Rossi's Journal of Nuclear Physics has published online a paper of the author, entitled "Stability of Light Nuclei", where the magnetic moments of several light nuclei are calculated, by considering that the interactions responsible for the nucleons equilibrium within the nuclei is due to the sort of interactions as

1. Spin-interactions between nucleons captured by the central $2 \mathrm{He} 4$. It occurs between deuteron-deuteron, deuteron-proton, deuteron-neutron, proton-proton, proton-neutron, and neutronneutron. Deuterons have the maximum interaction with deuterons, since in total there is interaction of four fields. But the best spin-interaction is deuteron-neutron, because there is no Coulomb repulsion between them, and the centrifugal force on the neutron is weak, and because there are three fields interacting, two of neutrons and one of the proton. Obviously the spin-interaction between deuteron and proton is weaker, because of the Coulomb repulsion. So, there are seven intensities of spin-interaction, as follows; 1) very weak, when two fields (protons-proton, proton-neutron, or neutronneutron) interact with contrary spins; 2) weak, when two fields interact with parallel spins; 3) medium, when three fields (of one deuteron plus a proton, or neutron) interact with contrary spins; 4) strong, when three fields interact with parallel spins, which is $40 \%$ stronger than the medium interaction; 5) very strong, when two deuterons interact with contrary spins; 6) maximum, when two deuterons interact with parallel spins; 7) when two deuterons interact with the central $2 \mathrm{He} 4$ with perfect symmetry, and it occurs only in the $4 B e 8$, as will be shown later.

2. Gravito-electriciton force acting on deuterons, protons, and neutrons. Such force is produced because protons and neutrons are crossed by the n(o)-flux of the central $2 \mathrm{He} 4$ (the rotation of the quarks, around the n(o)-flux of $2 \mathrm{He} 4$, produces the interaction responsible for this force). Let us call them protonn(o)-flux and neutron-n(o)-flux forces (or nucleon-n(o)-flux force, when it is not specified whether it is proton or neutron). Such force obliges a nucleon to change its spin when it moves from inner to outer side (or vice versa) of Ana and Douglas. 
3. Magnetic force of attraction between the proton and the central $2 \mathrm{He} 4$, due to the rotation of the proton charge around it. As the neutron has no charge, there is no magnetic force on it.

4. Spin-interaction force between nucleons and the central $2 \mathrm{He}$. Such force, alone, is no able to keep a nucleon bound to the central $2 \mathrm{He} 4$, because it is weaker than the centrifugal force. Coulomb and centrifugal forces are counterbalanced by three forces acting together: spin-interaction, nucleon-n(o)flux, and magnetic ones. As on a lonely neutron does not act the magnetic force, only the spin-interaction with the central $2 \mathrm{He} 4$ (acting together with neutron-n(o)-flux) is unable to counterbalance the centrifugal force on it, and neutron is expelled, as occurs, for instance, in $2 \mathrm{He} 5$ and $3 \mathrm{Li} 5$.

5. Considering the interaction of a proton with the central $2 \mathrm{He} 4$, the proton is submitted to the dispute between centrifugal force, acting together with Coulomb force, against the magnetic force acting together with proton-n(o)-flux force and with proton $\leftrightarrows 2 \mathrm{He} 4$ spin-interaction force.

6. For the sake of calculation of nuclear magnetic moments, we may consider that Coulomb repulsion on the proton is counterbalanced by proton $\leftrightarrows 2 \mathrm{He} 4$ spin-interaction acting with proton-n(o)-flux force. Thereby there is no need to consider any of them. And the calculus involves only the equilibrium between magnetic force and centrifugal force.

By considering that nucleons are bound by the interactions above, it is possible to explain why, after the decay of the halo neutron in $4 B e 11$, the newborn proton (far away $7 \mathrm{fm}$ from the core of the newborn 5B11), moves back to the core, instead of being expelled by Coulomb repulsion, as is expected by considering any current nuclear model where nucleons are bound via strong nuclear force. And the reason is because, along the 13.8 seconds of $B e 11$ lifetime, the orbit radius of the halo neutron grows up to $7 \mathrm{fm}$ faraway of the core. The orbit radius increases because the centrifugal force is a little stronger than the sum of spin-interaction force (with the core) acting together with neutron-n(o)-flux force. When the halo neutron arrives to the place $7 \mathrm{fm}$ far away to the core, it decays, and on the newborn proton appears an additional magnetic force pulling it toward the core. As already said, within distances in the range of few femtometers, Coulomb forces do not follow the well-known equation $F=K\left(Q q / d^{2}\right)$, as shown in (Guglinski, 2018b), but actually they are very weaker than nuclear theorists suppose, and thereby the electric repulsion is weaker than the magnetic attraction, and unable to prevent the return of the newborn proton to the core of the newborn $5 B 11$. NOTE: for the calculation of the magnetic moments of light nuclei in the paper Stability of Light Nuclei, the isotopes 3 Li6 and $3 \mathrm{Li7}$ were used for the calculation of some initial parameters. However, in 2013 the author did not discover yet the mechanism responsible for the rotation of the nuclei, and then in that first attempt he had considered that $3 \mathrm{LiC}$ and $3 \mathrm{LiT}$ rotate with the same angular velocity. But $3 \mathrm{Li6}$ and 3 Li7rotate with different angular velocities, which are calculated in a paper to be published in upcoming September. At that paper are introduced the principles and laws governing the mechanism that produces the revolutions of all nuclei, and are calculated (with very good accuracy), the magnetic moments of several isotopes whose number of protons $\mathrm{Z}$ varies between 3 and 30.

\section{WHY MIRRORS $3 L i 7$ AND $4 B e 7$ HAVE NOT CLOSE VALUES OF ELECTRIC QUADUPOLE MOMENTS?}

Other fundamental question is related to the electric quadrupole moment for $4 B e 7$. Figure 10 shows how the nucleons must be distributed in the mirror nuclei $3 \mathrm{Li7}$ and $4 B e 7$, as we have to expect from the principles of the SNP. Experiments have measured $Q(L i 7)=-0.406$ barns. But the value of $Q(B e 7)$ is not quoted in any nuclear table, and the question, is why? Looking at the structures of $3 \mathrm{Li7}$ and $4 \mathrm{Be} 7$ shown in Figure 10, it is hard to understand why $Q(\mathrm{Li7})$ can be measured, but there is no way to measure $Q(B e 7)$. After all, they are mirror nuclei, and lifetime of $4 B e 7$ is 53 days.

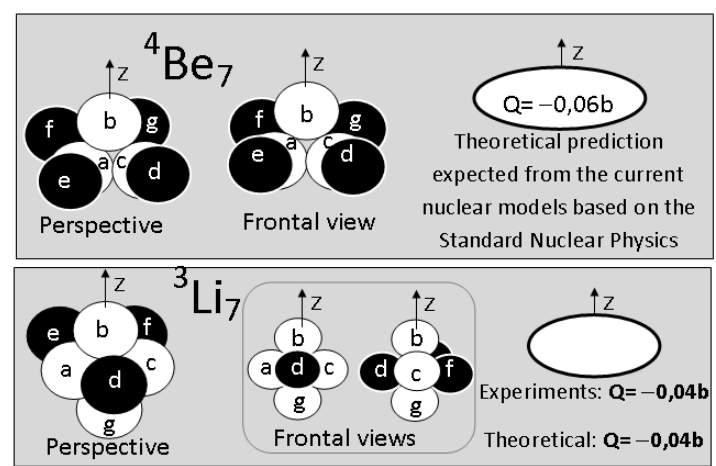

Fig 10. Electric quadrupole moments of $3 \mathrm{LiT}$ and $4 \mathrm{Be} 7$

The structure of $3 \mathrm{Li7}$, according to the Hexagonal Floors model, is shown in Figure 11. Only charged nucleons, as protons and deuterons, have successful capture by the $\mathrm{n}(\mathrm{o})$ flux generated by the central $2 \mathrm{He} 4$. Lonely neutrons cannot be captured by the n(o)-flux (they must be bound to deuterons via spin-interaction). In Figure 11 , beyond $F_{S i}$ and $F_{G E}$ (spininteraction and gravito-electriciton forces of attraction with the central $2 \mathrm{He} 4$ ), the lonely neutron $\mathrm{n}-3$ is bound to the deuteron (formed by proton $p 1$ and neutron $n 2$ ), through their spininteraction attraction (note that the deuteron and the neutron have up-spin). Because the duo $F_{S i}$ and $F_{G E}$ is not able of binding a neutron to the central $2 \mathrm{He} 4$, this is the reason why any helium nucleus with $\mathrm{N}>2(2 \mathrm{He} 5,2 \mathrm{He} 6,2 \mathrm{He}, 2 \mathrm{He} 8 \ldots)$ is no stable, because the neutron is expelled by the centrifugal force. For instance, consider that a nucleus $2 \mathrm{He} 4$ captures a proton, and they form the $3 \mathrm{Li}$. The centrifugal force on the proton in the $3 L i 5$ is half of the centrifugal force on the deuteron in $3 \mathrm{Li6}$. But the magnetic force on the proton of the $3 \mathrm{Li} 5$ is the same of the magnetic force on the deuteron of the 3 Li6, because the charge of proton and deuteron is the same. So, the centrifugal force, on the proton in $3 \mathrm{Li} 5$, is no able to counterbalance the attraction force between the proton and the central $2 \mathrm{He} 4$, and the proton falls inside the $2 \mathrm{He} 4$, and the $3 \mathrm{Li} 5$ decays, ejecting the proton.

At the ground state, the $2 \mathrm{He} 4$ does not rotate. But when it captures a nucleon (a neutron, a proton, or a deuteron), immediately the newborn nucleus starts to rotate. For instance, if $2 \mathrm{He} 4$ captures a neutron, the newborn $2 \mathrm{He} 5$ starts to rotate, and the neutron is expelled by the centrifugal force. The mechanism responsible for the rotation is explained in the paper "Calculation of magnetic moments of light nuclei with number of protons between $Z=3$ and $Z=30$ ". 


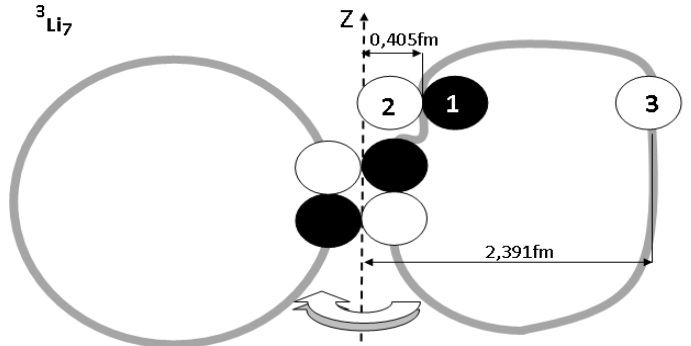

Fig 11. The nucleus $3 L i 7$, where the neutron number 3 is bound via spin-interaction with the deuteron formed by proton 1 and neutron 2

According to Hexagonal Floors Model, the value of $Q(B e 7)$ must be very close to zero, and we realize why by looking to the structure for $\mathrm{Be} 7$ shown in Figures 12 and 13. Figure 12 shows that $p 1$ proton forms a deuteron with $n 2$ neutron, and the deuteron is captured by the string of gravitons produced by the central $2 \mathrm{He} 4$. Also $\mathrm{p} 3$ proton is captured by the string.

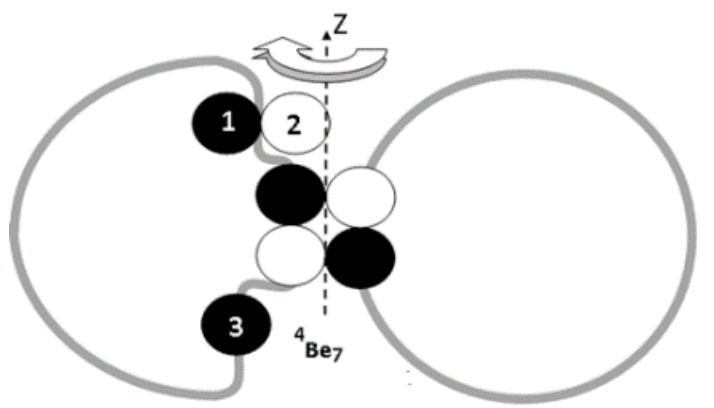

Fig 12. Distribution of nucleons in the $4 B e 7$, according to the Hexagonal Floors Model.

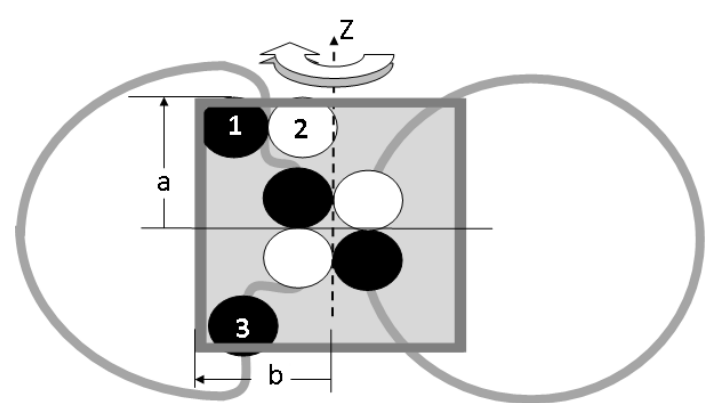

Fig 13. Having "a" and "b" approximately the same length, the value of $\mathrm{Q}$ for $4 \mathrm{Be} 7$ must be very near to zero.

Figure 13 shows that the distance between $p 1$ proton and the center of the central $2 \mathrm{He} 4$ is "a", whereas $\mathrm{p} 3$ proton has a distance " $b$ " to the center, and "a" and "b" have approximately the same length. Thereby, due to the rotation of the nucleus, the charges of $p 1$ and $p 3$ occupy a volume which is approximately of a cube, in order that $Q(B e 7)$ is very close to zero. Actually "a" is a little longer than "b", as we will see later.

\section{WHY IS THE ELECTRIC QUADRUPOLE MOMENT FOR $4 B e 7$ MISSING IN NUCLEAR TABLES?}

The author exchanged several emails with many experimentalists involved in the work of measuring the value for $Q(B e 7)$, including Dr. Nicholas Stone, editor of the nuclear table published by Clarendon Laboratory. The author hoped to discover why the value for $Q(B e 7)$ is not quoted in the nuclear tables, because he had the suspicion that several experimentalists already had tried to measure it, but as the experiments obstinately have always refused to supply any result far away from zero, the all the experimentalists have supposed that something wrong occurred in the process of measurement, and they decided do not send any report to any editor of nuclear table.

The physicists allege that $Q(B e 7)$ is not quoted in nuclear tables because it was never measured, but such argument makes no sense, because,

1- $\mathrm{Li7}$ and $\mathrm{Be} 7$ are mirror isotopes, and therefore they should have similar structures (according to $\mathrm{SNP}$ ). And as $\mathrm{Q}\left(\mathrm{Li}_{7}\right)=$ -0.0406 barns was measured, then why $Q(B e 7)$ was not?

2- Along 20 years some theorists have emphasized the importance of getting $Q(B e 7)$ measurement. John Bahcall called the attention to the importance of the measurements of the quadrupole moments for $B e 7$, because it is crucial for the understanding of the sun shines by nuclear fusion reactions among light elements in its interior (according to the current foundations of the SNP, of course). In the paper he says,

A measurement of the $B e^{7}$ quadrupole moment would help to distinguish between different nuclear models for the $\mathrm{Be}^{7}(\mathrm{p}, \gamma) \mathrm{B}^{8}$ reaction (Bahcall, 1998).

3- The crucial importance of $Q(B e 7)$ measurement, for the confirmation of the theory of nuclear synthesis in the Sun, was emphasized in several papers, as

a -We find that the low-energy astrophysical $\mathrm{S}$-factor is linearly correlated with the quadrupole moment of $7 \mathrm{Be}$. A range of parameters is found where the most important $7 \mathrm{Be}$ and $7 \mathrm{Li}$ properties are reproduced simultaneously. Despite extensive experimental efforts, the $\mathrm{Be}^{7}(\mathrm{p}, \gamma) \mathrm{B}^{8}$ cross section is still the most uncertain nuclear input to the standard solar model, due to a significant spread among the values of $S 17$ deduced from the various experiments. For a set of parameters that reproduce simultaneously the most important properties of $7 B e, 7 L i$, and $8 B$, we predict $Q 7 B e$ to be between $-6.0 \mathrm{efm}^{2}$ and $-6.9 \mathrm{efm}^{2}$.

b -We also note that a precise measurement of the ${ }^{7} \mathrm{Be}$ quadrupole moment or radius would test the self-consistency of our conclusions (Csótó, Langanke, Koonin, \& Shoppa, 1995).

4- The theoretical $Q(B e 7)$ was predicted to be between $-6 \mathrm{fm}^{2}$ and $-7 \mathrm{fm}^{2}$, as Csoto \& Langanke write in the page 5 of their paper, We note again that a measurement of the ${ }^{7} \mathrm{Be}$ quadrupole moment would place some additional constraints on the consistency of our calculations. For the complete $\mathrm{He}^{4}+$ $\mathrm{He}^{3}+p$ model calculation the simultaneous reproduction of the indicators predict $Q_{7}$ to be in the range $-(5: 5-$ 6: 0$) \mathrm{efm}^{2}$. However, this value is smaller than the one $\left(Q_{7}=\right.$ $-6.9 \mathrm{efm}^{2}$ [9]) obtained if we chose the cluster size parameters such to reproduce the quadrupole moment of the analog nucleus $7 \mathrm{Li}$. Does this already point to the necessity of a further enlargement of the model space beyond the $\mathrm{He}^{4}+$ $\mathrm{He}^{3}+\mathrm{p}$ three-cluster model which would then also effect our results obtained for $B e^{7}$, e.g., change the $B e^{7}$ quadrupole moment? (Csótó \& Langanke, 1998).

Theirs conclusion is that $Q(B e 7)$ is between -0.06 and 0.069 barns, a value close to $Q(L i 7)=-0.04 b$, and such value of $Q(B e 7)$ predicted by them comes from the fact that they consider two similar structures for $\mathrm{Li7}$ and $\mathrm{Be} 7$.

5- But as the measurement of $Q(B e 7)$ is so important for the confirmation of the theory of nuclear synthesis in the Sun, a question arises: why, along more than 40 years, did not the 
experimentalists succeed to measure it, and to get a value at least close to $Q(\mathrm{Li7})=-0.04$ barns?

6- In 2013 the author sent an email to Dr. Nicholas Stone, saying the following: Dear Dr. Stone, I suspect that $B e 7$ has $\mathrm{Q}$ very near to zero, that's why it is not quoted in nuclear tables. I suspect that the experimentalists already had tried to measure it, and they had expected to measure a value near to the value of $\mathrm{Q}$ for $9 \mathrm{Be}$ ( $0.053 \mathrm{barns})$. As the experimentalists did not succeed to get a value far away from zero (and as they know that from the theoretical viewpoint $B e 7$ cannot have $Q$ very near to zero) then they did not report the results of experiments. Dr. Attila Csoto had calculated theoretically that $Q(B e 7)$ must be in order of 0.07 barns. I have my suspicion because 1) $B e 9$ is stable. 2) $B e 7$ has half-life of 53 days. 3) Both them are very small. 4) $Q(B e 9)$ had been measured by atomic beam, and got 0.053 barns. AS CONCLUSION, why cannot the Q for $B e 7$ be measured by atomic beam? So, I suspect that $\mathrm{Q}$ for $B e 7$ had already been measured by atomic beam (like done for $9 \mathrm{Be}$ ), but the experiments had NOT detected a value far away of zero (as expected theoretically). Do you think that my suspicion can explain why $\mathrm{Q}$ for $B e 7$ is not quoted in nuclear tables?

Dr. Stone sent the following reply: Just to say that, IF a measurement had been made, it would certainly have been published. However, perhaps Dr. Stone was wrong, because we cannot be sure whether the experimentalists were not afraid to report a value very close to zero, and the reason is shown ahead.

A) Values equal to zero are not quoted in nuclear tables. For instance, magnetic moments for even-even nuclei are not quoted, because they are null. Then, in the case the experimentalists have measured a value very close to zero, then they did not report it to the editors of nuclear tables.

B) $Q($ Li6 $)=-0.0008$ barns a very small value, and is quoted in nuclear tables. But suppose that $Q(L i 6)$ is very biggest than $Q(B e 7)$, which therefore is very, very small. In this case an experimentalist would be afraid to report to editors of nuclear tables such a very, very small value, because he could be afraid that he made some error in the procedure of measurement. Our final conclusion about the subject seems to be unavoidable; the electric quadrupole for $B e 7$ denies the foundations of the SNP.

\section{PUZZLE OF THE $4 B e 8$ INSTABILITY AND THE INCOMPATIBILITY BETWEEN PAULING'S THEORY AND THE STRONG NUCLEAR FORCE}

For the nuclear theorists, a measurement of $Q(B e 7)$ is of interest, because it would help distinguish between different nuclear models for the $B e 7$ nucleus. It means that they do not know how is the structure of Be7. And so they also do not know how is the structure of $B e 8$ (before its decay).

By considering that nuclei are bound via strong nuclear force, as is considered in all the current nuclear models, it is impossible to find any physical cause responsible for the instability of $B e 8$. After all, as protons and neutrons are bound via strong force, and $B e 7$ lifetime is 53 days, one neutron added to $B e 7$ would have to result in a stable $B e 8$, where the fourth neutron would be bound via strong nuclear force to the $B e 7$. And other puzzle comes from the fact that all the light even-even nuclei with $Z=N$ are stable, except $4 B e 8$. Why? Linus Pauling proposed some "rules", and according to his theory there must be present a three nucleon cluster, either triton [NPN] or [PNP], rotating against either: (1) another three nucleon cluster or (2) a helium-4 cluster for any isotope with $A>4$ to be "stable". Hence, Be8, being composed of two helium-4 rotating clusters [PNPN] [PNPN] violates this basic "rule of assembly" dynamic and is thus "unstable".

However, Pauling's rule is no satisfactory. First of all because his theory simply explains nothing. He had observed what are the stable light nuclei in nature, and had proposed rules based on that observation. He had proposed that $B e 8$ is formed by two helium-4. But if $\mathrm{Be} 8$ structure is $\mathrm{He} 4+\mathrm{He} 4$, then $\mathrm{Be} 7$ structure must be $(\mathrm{He} 4+\mathrm{He} 3)$. However, $\mathrm{He} 4$ and $\mathrm{He} 3$ are stable, and thereby $\mathrm{Be} 7$ structure cannot be composed by helium- 4 and helium-3, because $B e 7$ is not stable, it decays in 53 days. As consequence, $B e 8$ structure cannot be composed by two helium-4. So, Pauling's theory is unacceptable (when we consider his theory under the laws of the SNP). On another hand, let us analyze $B e 8$ structure by considering that nucleons are bound via strong nuclear force, as considered in the SNP. Looking at the $B e 7$ structure in Figure 10, we realize that the four protons have to take those places (surrounding the three neutrons in the center of the nucleus) because according to the SNP there is a very strong Coulomb repulsion between the protons, and this is the reason why they hide themselves behind the neutrons. Thereby, by considering the laws of the $\mathrm{SNP}$, the $B e 8$ structure would have to be as shown in Figure 14 , where the four protons hide themselves behind the central cluster formed by neutrons.

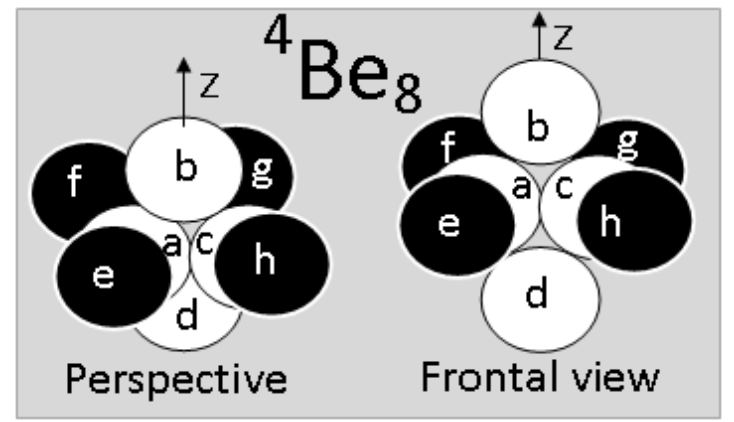

Fig 14. Be8 structure, as we have to expect from the laws of interactions according to the Standard Nuclear Physics

Consider the assumption that nucleons are bound via strong nuclear force together with Pauling's theory (that $B e 8$ is composed by two He4). In such a way, Be8 structure shown in Figure 14 should evolve as shown in Figure 15, where the central He4 is formed by fusion of protons "e" and " $h$ " with neutrons "a" and "c". As the He4 occupies the center of $B e 8$, then protons "f" and " $g$ " have to take one among the three opposite positions shown in (A), (B ), or (C), in Figure 15, because they are under very strong Coulomb repulsion, and thereby the second helium-4 cannot be formed, and Pauling theory fails.

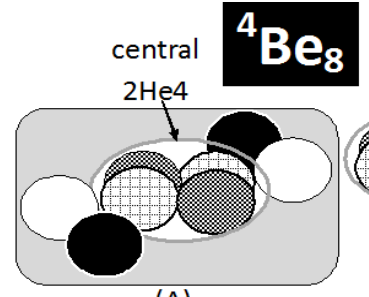

(A)

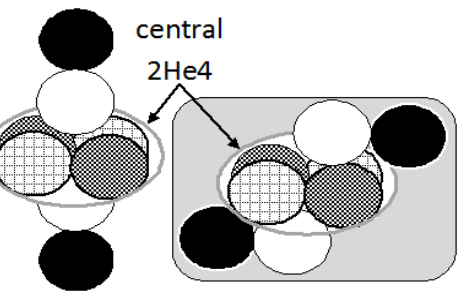

(C)
Fig 15. How must be $B e 8$ structure, according the laws of the Standard Nuclear Physics, by considering Linus Pauling's rules 
An interesting fact must be noted about Pauling's theory. Because while his theory is unacceptable if we consider that nucleons are bound via strong nuclear force, on another hand his theory makes sense if we consider it under the laws of the new Hexagonal Floors model. Indeed, as we will see ahead, when $B e 8$ is formed, the two deuterons (captured by the $\mathrm{n}(\mathrm{o})$ flux of the central $2 \mathrm{He} 4$ ), meet together and fuse, and they form a second $2 \mathrm{He} 4$ (and this second $2 \mathrm{He} 4$ cannot be captured by $\mathrm{n}(\mathrm{o})$-flux of the central $2 \mathrm{He} 4)$. Before to understand why $B e 8$ is instable, there is need to understand how nucleons move when they are captured by the central $\mathrm{He} 4$ of light nuclei.

\section{NUCLEONS CAPTURED BY THE n(o)-FLUX}

Figure 16 shows what happens with nucleons when they are captured by the n(o)-flux. The central field generated by the central $2 \mathrm{He} 4$ is divided in two sides: Douglas and Ana. And each one of them has two sides: inner and outer. The magnetic moment of a nucleon captured by n(o)-flux depends on 1- If the direction of the $\mathrm{n}(\mathrm{o})$-flux coincides with the direction of the vector-spin of the nucleon, the sign of the magnetic moment does not change

2- If the direction of the $\mathrm{n}(\mathrm{o})$-flux and the vector-spin of the nucleon have contrary direction, the sign of the magnetic moment is changed

3- These two laws are resulting from the mechanisms of interaction between the $\mathrm{n}(\mathrm{o})$-flux of the nucleon and the $\mathrm{n}(\mathrm{o})$ flux of the central $2 \mathrm{He} 4$. For instance, the magnetic moment of the proton, measured by experiments, is $+2.793 \mu N$, referred to an up-spin. Inside a nucleus, if a proton is crossed by a $\mathrm{n}(\mathrm{o})$ flux moving in the same direction of its vector-spin, its magnetic moment continues being $+2.793 \mu N$. But if the direction of the n(o)-flux is contrary to the direction of the proton vector-spin, then the magnetic moment, of that proton inside the nucleus, becomes negative, $-2.793 \mu N$.

The same happens regarding the neutron and the deuteron. For instance, in $3 \mathrm{Li7}$ the deuteron contributes with $\mu=+0.857 \mu \mathrm{N}$, because its up-spin-vector has the same direction of the up$\mathrm{n}(\mathrm{o})$-flux (in the inner side of Douglas), while the neutron contributes with $\mu=-(-1.913)=+1.913 \mu N$, because its upspin-vector has contrary direction of the down-n(o)-flux (in the outer side of Douglas). See the Li7 structure in the Figure 30. In Figure 16, the sign of the magnetic moment is related to North and South poles, in analogy with the poles $\mathbf{N}$ and $\mathbf{S}$ of a magnet. If a nucleon has upside North pole, it has positive magnetic moment. If South pole is upside, magnetic moment is negative. Note that in the top of Figure 16 there is a rectangle with the title "Central $2 \mathrm{He} 4$ ", showing how protons and neutrons (of the central $2 \mathrm{He} 4$ ) have interaction. From that rectangle, we conclude that the four nucleons, of the central $2 \mathrm{He} 4$, interact with maximum binding energy, possible for the interaction between four nucleons. Looking at the proton structure shown in Figure 9, we realize that the up-n(o)-flux produced by the proton of the central $2 \mathrm{He} 4$ (in the side Douglas, see Figure 16), is generated by two up quarks with up-spin (of that proton), and one down quark with down-spin. This means that the most "comfortable" place, for the proton to be captured, is by two sort of up-n(o)-fluxes, as

A) In the Douglas side, with up-spin in the inner side, and with down-spin in the outer side, and

B) In the Ana side, with down-spin in the inner side, and with up-spin in the outer side.
Concerning the neutron, as it has two protons with contrary spins, they have no influence for the most comfortable position for the neutron in the n(o)-flux. However, inside the neutron, the down quark has up-spin (contrary to its spin in the structure of the proton, see Figure 9), and therefore the most comfortable place for the neutron is being captured by down$\mathrm{n}$ (o)-fluxes (contrary to the comfortable positions for the proton).

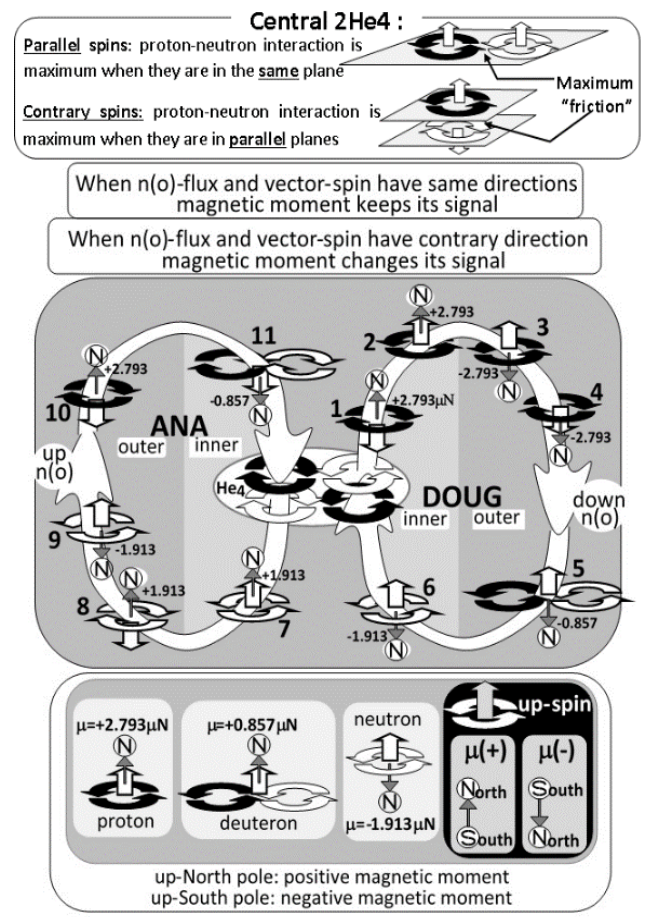

Fig 16. How n(o)-flux influences the magnetic moments of protons, neutrons, and deuterons captured by the central $2 \mathrm{He} 4$

3 Li6 is stable because magnetic and centrifugal forces on the deuteron (captured by the n(o)-flux of the central 2 $\mathrm{He} 4$ ) are in equilibrium. As already explained, the sum of other repulsion and attraction forces (Coulomb repulsion and spin-interaction, acting together with nucleon-n(o)-flux interaction) cancel each other. Lifetime of $3 L i 5$ is $3.7 \times 10^{-22} s$. It decays so quickly because the lonely proton in $3 \mathrm{Li} 5$ and the deuteron in 3 Li6have the same magnetic force attraction with the central $2 \mathrm{He} 4$, but the centrifugal force on the lonely proton is half of that in the deuteron, and so the proton of $3 \mathrm{Li} 5$ falls quickly into the central $2 \mathrm{He} 4$. Figure 17 shows the $\mathrm{He} 5$ decay and the reaction $L i_{5}+$ $p \rightarrow B e_{6}$.

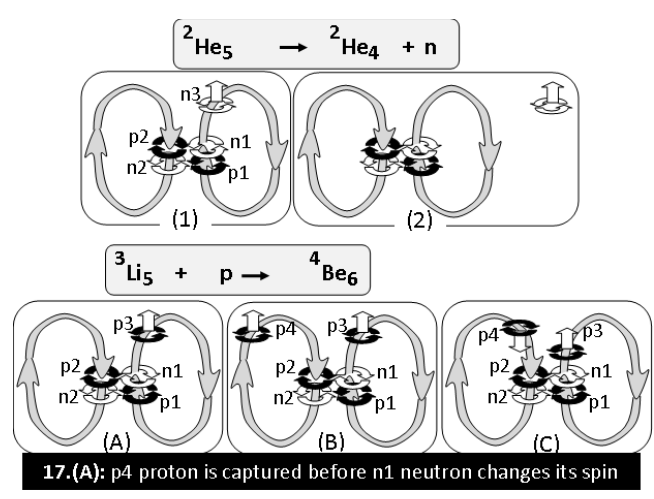

Fig 17. How nucleons move inside the light nuclei when they captured by the $n(0)$-flux 
In 17.(1) is shown the structure of the $2 \mathrm{He} 5$. Before the capture of $n 3$ neutron, $n 1$ neutron and $p 2$ proton are aligned with down-spin. As is up-n(o)-flux in the inner side of Douglas, then $n 3$ neutron is captured with up-spin. Being between $n 3$ and $p 1$ (both with up-spin), $n 1$ neutron changes its spin from down to up, and $\mathrm{He} 5$ gets spin 3/2. In 17.(2) it is seen that $n 3$ is expelled by centrifugal force. In 17.(A) it's shown $3 \mathrm{Li5}$ structure. Before the capture of the proton $\mathrm{p3}$, central $2 \mathrm{He} 4$ has $n 1$ neutron and $p 2$ proton aligned with down-spin. When $p 3$ proton is captured and it approaches to the central $2 \mathrm{He} 4$, as $p 3$ and $p 1$ have up-spin, and $n 1$ is between them, then $n 1$ abandons its interaction with $p 2$, getting up-spin together with $p 1$ and $p 3$, and $3 L i 5$ gets spin $3 / 2$. Magnetic moment for $3 L i 5$ is not quoted in nuclear tables, and the reason, perhaps, is because it has two magnetic moments, before and after changing the spin of $n 1$ neutron. Since its lifetime is very short, in order of $10^{-22} \mathrm{~s}$, there is practically an overlap of the two magnetic moments, before and after $n 1$ changes its spin. Then $p 3$ proton falls into the central $2 \mathrm{He} 4$ and is expelled by Coulomb repulsion along $\mathrm{z}$-axis direction (because, when the center of $p 3$ proton becomes coincident with the $\mathrm{z}$-axis, it stops to rotate about the central $2 \mathrm{He} 4$, and so becomes null the magnetic force on $p 3$, and it is expelled by electric repulsion).

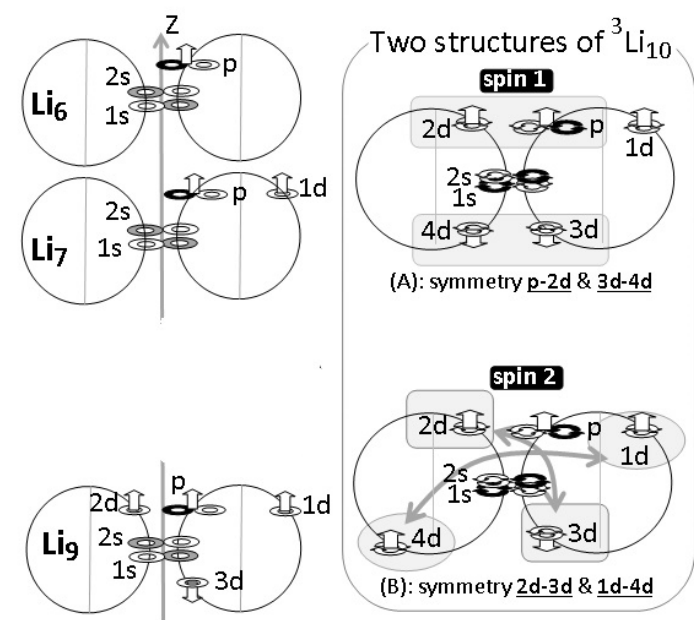

Fig 18. The reason why 3 Li10 has two different structures

Note: as happens with $3 L i 5$, whose magnetic moment is not quoted in nuclear tables, because probably it has two structures with different spins, magnetic moment of $3 \mathrm{Li10}$ also is not quoted, because it has two different structures, one with spin 1 and other with spin 2 . Figure 18 shows why 3 Li10 has two structures.

In the left side is shown how the energy levels s, p, and d, of the nucleons, are distributed since $3 \mathrm{Li6}$ till $3 \mathrm{Li}$. Note that in $3 L i 10$, the neutron which occupies the last level $4 d$, can take two different positions, and each one of the positions is able to promote a symmetric structure for the $3 \mathrm{Li10}$, as one with spin 1 , and other with spin 2 . Now let us continuing to analyze what happens with 3 Li5 in Figure 17.(A), by supposing that (in the first $1.0 \times 10^{-23}$ second of its lifetime, before $n 1$ neutron changes its spin) $3 \mathrm{Li} 5$ captures $p 4$ proton, and they form $4 \mathrm{Be} 6$, with spin 2, whose lifetime is $50 \times 10^{-23} \mathrm{~s}$. So, with the capture of a proton, the newborn Be6 has gained more $46.3 \times 10^{-23} \mathrm{~s}$ of life. Let us see why the lifetime has increased, by looking in the Figure 17.(B), where it is seen that $3 \mathrm{Li} 5$ captures the fourth $p 4$ proton, with up-spin, in the outer side of Ana, because while the Coulomb force tries to keep $p 3$ and $p 4$ very far away, however they have spin-interaction (promoted by the "friction" of the n(o)-fluxes of the two protons with up-spin). In 17.(C) it's seen that, as the magnetic attraction between the central $2 \mathrm{He} 4$ and each of $p 3$ and $p 4$ protons (acting together with their spin-interaction) is stronger than their Coulomb repulsion, then $p 4$ proton moves to the inner side of Ana and changes its spin, and so Be6 gets spin zero, while $p 3$ continues going toward the direction of the central $2 \mathrm{He} 4$. Firstly, $p 3$ falls into the $2 \mathrm{He} 4$, followed by $\mathrm{p} 4$, and they have the same fate of $p 3$ proton in the decay of $2 \mathrm{He} 5$, that is, $p 3$ and $p 4$ leave the $2 \mathrm{He} 4$ along z-axis.

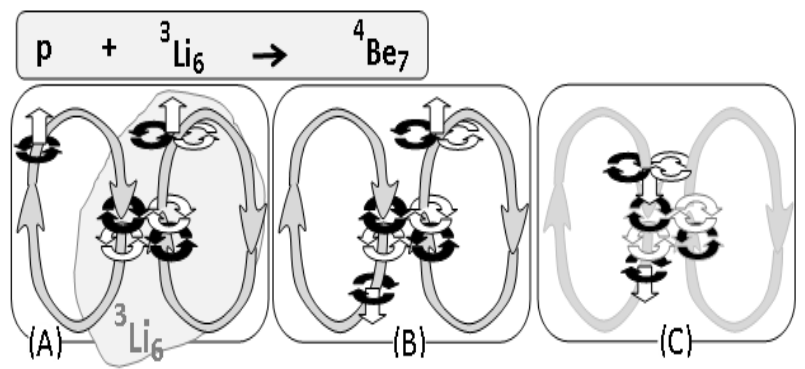

Fig 19. Formation of $4 \mathrm{Be} 7$ by capture of proton by $3 \mathrm{Li6}$

A similar mechanism occurs with $4 B e 8$, except that centrifugal force on the deuterons in $4 B e 8$ is twice than in protons of $4 \mathrm{Be} 6$. But before seeing how occurs $B e 8$ decay, let us see how nucleons move when a proton is captured by 3 Li6 and they form $4 B e 7$, as shown in Figure 19. The deuteron in 3 Li6 occupies the inner side of Douglas, as seen in 19.(A). We already know that the proton will be captured with up-spin, by the up-n(o)-flux in the outer side of Ana, as consequence of its interaction with the deuteron of $3 \mathrm{Li6}$.

Due to Coulomb repulsion, the proton is pushed to the position shown in 19.(B), because, as the deuteron is closer to the central $2 \mathrm{He} 4$, it is strongly tied to the $2 \mathrm{He} 4$. But when the proton arrives to that position shown in (B), it becomes strongly tied to the central $2 \mathrm{He} 4$, because the centrifugal force on the deuteron is twice of that on the proton. As consequence the deuteron is pulled to the inner Ana side thanks to its spininteraction with the proton, and the deuteron aligns its spin with the proton, as shown in (C), and $B e 7$ keeps that structure along 53 days, before decaying.

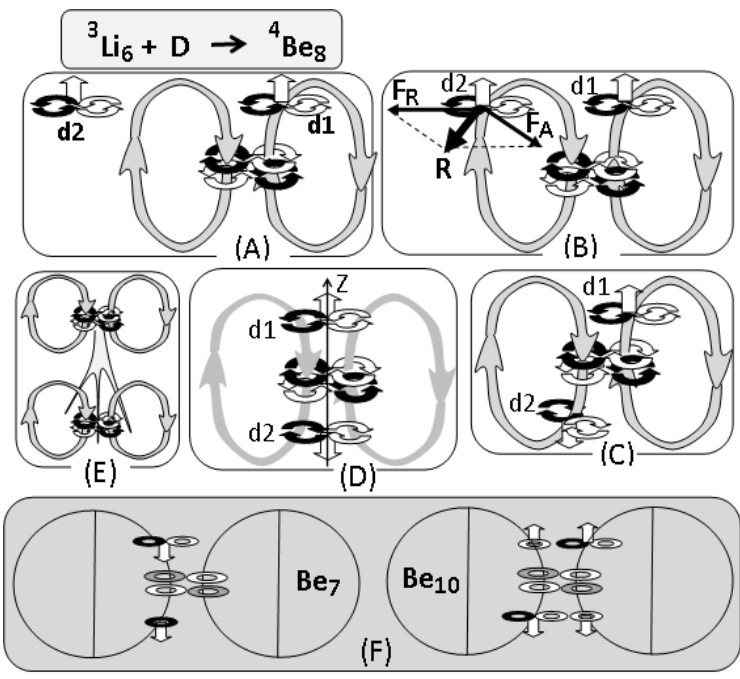

Fig 20. Why $4 B e 8$ is unstable 
Finally, let us analyze Be8. Figure 20.(A) shows a nucleus 3 Li6, and a free deuteron going in its direction. In 20.(B) it's shown that deuteron $d 2$ is captured with up-spin in the outer side of Ana, and they form $B e 8$ with spin 2 . Due to Coulomb repulsion between $d 2$ with the charges of $d 1$ and the central $2 \mathrm{He} 4$, acting together with centrifugal force pushing $d 2$, and because $d 1$ is strongly attached to the central $2 \mathrm{He} 4$ by spininteraction, then $d 2$ is quickly pushed, and goes to the position shown in 20.(C), and the $B e 8$ gets the spin zero. Note that in their positions shown in 20.(C), $d 1$ has maximum spininteraction with the upper side of the central $2 \mathrm{He} 4$, and $d 2$ has maximum spin-interaction with the lowest side of the central $2 \mathrm{He} 4$. So, the central $2 \mathrm{He} 4$ promotes the alignment of $d 1$ and $d 2$, by putting their centers coincident with the $\mathrm{z}$-axis of the central $2 \mathrm{He} 4$, as shown in 20.(D). Due to Coulomb repulsions, $d 1$ and $d 2$ are submitted to a zig-zag oscillatory motion along the $\mathrm{z}$-axis, while at the same time they are submitted to the maximum spin-interaction force with the central $2 \mathrm{He} 4$, pulling them one against the other. They meet together, and fuse transmuting to helium-4, as shown in 20.(E). This phenomenon of alignment of two nucleons, promoted by the central $2 \mathrm{He} 4$, occurs only with two deuterons, as consequence of the perfect symmetry regarding the central $2 \mathrm{He} 4$. While in $4 B e 8$ the central positions shown in 20.(C) is occupied by a duo of deuterons, in $4 B e 7$ the central position is occupied by a duo deuteron-proton, and thanks to such asymmetry $B e 7$ does not decay in fraction of seconds, as occurs with $B e 8$. And in $4 B e 10$ the central position is occupied by a duo (neutrondeuteron) and (deuteron-neutron), and, thanks to such asymmetry, $B e 10$ also does not decay in fraction of seconds (structures of $B e 7$ and $B e 10$ are shown in 20.(F) ).

\section{WHY HAVE SOME LIGHT NUCLEI SEVERAL DECAY MODES?}

As said, the interaction of the n(o)-flux with a unique lonely neutron is very weak, and it is not able to retain it bound to a light nucleus (lonely means that it is not bound to a deuteron via spin-interaction). However, of course the field of the neutron interacts with the fields of the nucleus, and this influences the time of the neutron emission. For instance, the lonely neutron in $2 \mathrm{He} 5$ has interaction with the four fields of two protons and two neutrons of the central $2 \mathrm{He} 4$, and it has lifetime $7 \times 10^{-23} \mathrm{~s}$. While the lifetime of $2 \mathrm{He} 7$ is $29 \times 10^{-23} \mathrm{~s}$, four times longer, because in $2 \mathrm{He} 7$ the two lonely neutrons interact one each other, and each one of them interacts with six fields. One interesting property of some light nuclei is their different decay modes. For instance, in $83.1 \%$ of decays $2 \mathrm{He} 8$ transmutes to $3 \mathrm{Li8}$, in $16 \%$ to $3 \mathrm{Li} 7$, and in $0.9 \%$ to $\mathrm{He} 5+\mathrm{H} 3$. And the question, is why?

First of all, let us see how neutrons are captured by the n(o)flux of the nuclei, as follows.

1- Concerning helium-4 isotopes, as they have no deuterons (with which a neutron could interact, so that they could be captured by the n(o)-flux in the inner sides of Ana or Douglas), and as neutrons have very weak interaction with the n(o)-flux, then they are preferentially (98\%) captured by the outer side of Douglas, or Ana. The reason is easy to understand, because, as the central $2 \mathrm{He} 4$ rotates, the strings which compose the $\mathrm{n}(\mathrm{o})$ flux have the faster speed in the periphery of the flux, where the radius $\mathrm{R}$ is maximum. As the speed of rotation $v=\omega . R$ of the strings is faster there, then obviously in that place occurs the stronger force interaction between the $n(0)$-flux of the central $2 \mathrm{He} 4$ and the n(o)-flux of the neutron to be captured. 2- Neutrons are captured by helium- 4 isotopes thanks to the rotation of the n(o)-flux, because there is interaction due to the speed of free neutrons (crossing the helium-4, coming from the space with rectilinear motion) and the speed of rotation of the strings, and also because, before being captured, as neutrons are moving in rectilinear motion, then they are not submitted to centrifugal force yet. But when a neutron is captured, as it has no magnetic attraction with the central $2 \mathrm{He} 4$, and its interaction with the string (which has captured it) is weaker than the centripetal force, the orbit radius of the neutron starts to dilate.

3- The stronger interaction occurs when the spin-vector of the neutron has the same direction of the n(o)-flux, and it is $60 \%$ stronger than if they have parallel directions. Therefore, neutrons with spin parallel to n(o)-flux direction have $60 \%$ more chance to be captured.

4- As it is very weak the spin-interaction between a lonely neutron with the n(o)-flux (as for instance in the 2He5), after its capture the radius orbit of the neutron starts to increase, because the centrifugal force is stronger. As the radius orbit increases, also gradually increases the centrifugal force $F_{C}=$ $m . \omega^{2} . R$, and so after some fractions of seconds the neutron leaves the nucleus.

Figure 21 ahead shows how the neutrons can be captured by $2 \mathrm{He} 4$, for the formation of the $2 \mathrm{He} 8$.
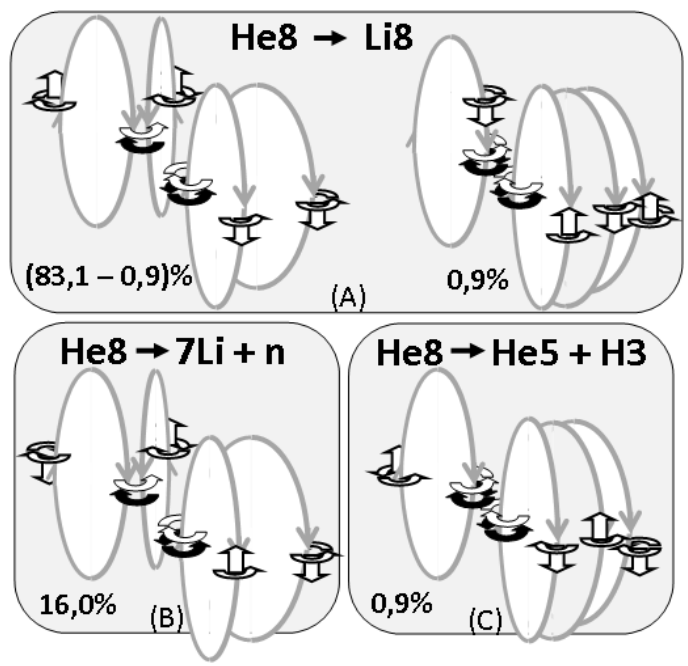

Fig 21. How neutrons of $2 \mathrm{He} 8$ are captured

In the sequence of Figures 22, 23, 24, and 25, it is shown how each combination of spins of the neutrons, in Figure 21, produce a specific $2 \mathrm{He} 8$ decay.

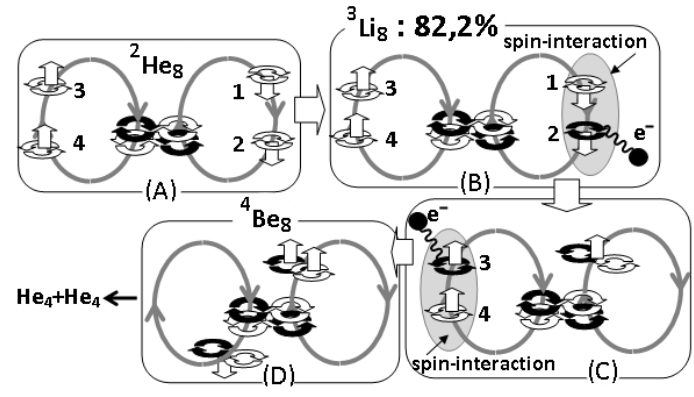

Fig 22. $\mathrm{He}_{8} \rightarrow \mathrm{Li}_{8}(82.2 \%) \rightarrow \mathrm{Be}_{8} \rightarrow \mathrm{He}_{4}+\mathrm{He}_{4}$ 


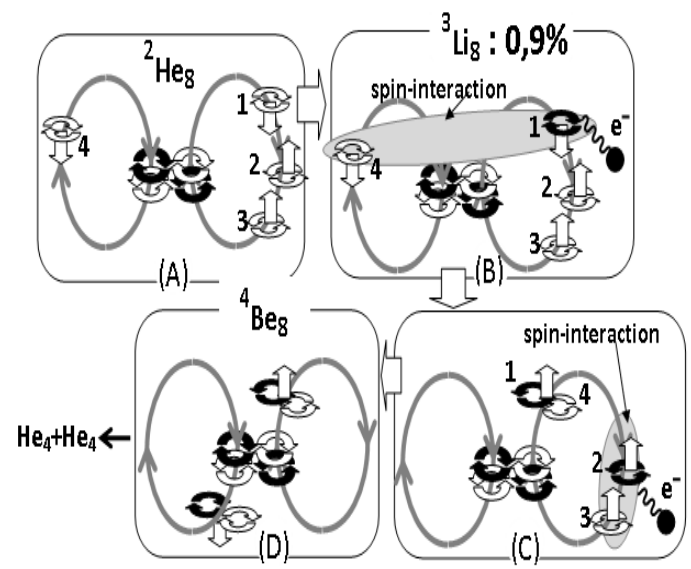

Fig 23. $\mathrm{He}_{8} \rightarrow \mathrm{Li}_{8}(0.9 \%) \rightarrow \mathrm{Be}_{8} \rightarrow \mathrm{He}_{4}+\mathrm{He}_{4}$

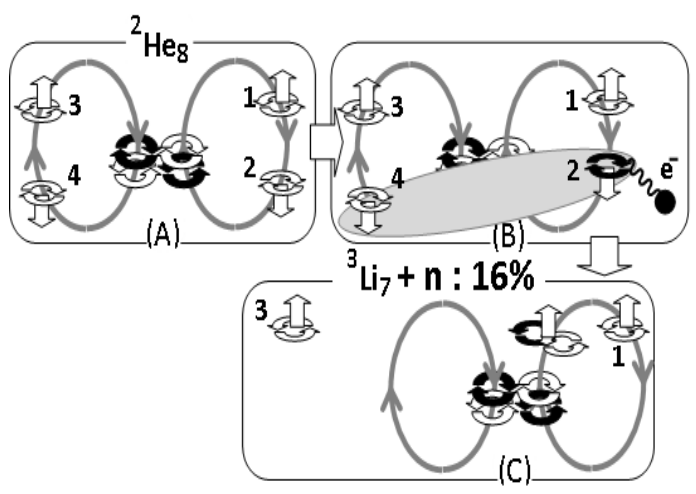

Fig 24. (16\%): $\mathrm{He}_{8} \rightarrow \mathrm{Li}_{7}+n$

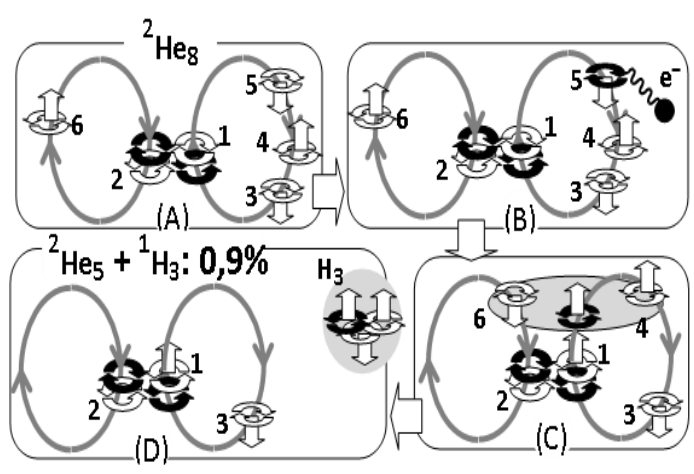

Fig 25. (0.9\%): $\mathrm{He}_{8} \rightarrow \mathrm{He}_{5}+\mathrm{H}_{3}$

In the Figure 25 we have, In (B)- neutron $n 5$ decays, and the newborn proton (under the magnetic force of attraction with the central 2He4), will move to the position shown in (C). In (C)- under interaction with the newborn proton, the neutron $n 1$ of the central $2 \mathrm{He} 4$ changes its spin, while neutrons $n 4$ and $n 6$ fuse with the newborn proton and they form $1 H 3$, which is expelled under the action of the centrifugal force.

\section{Li9 DECAY MODES AND ELECTRIC QUADRUPOLE MOMENT OF SOME LIGHT NUCLEI}

$L i 9$ structure is shown in Figure 26. By considering the values quoted in that structure, the value of magnetic moment calculated in is $+3.266 \mu N$, while the experimental is $+3.439 \mu N$. In Figure 30 are compared the structures of $L i 7, L i 9$, and $L i 11$, in order to understand the value of their electric quadrupole moments $Q\left(L i_{7}\right)=-0.040 b, Q\left(L_{11}\right)=-0.031 b$, and $Q\left(L i_{9}\right)=$ $0.025 b$ (sign no quoted in nuclear tables).

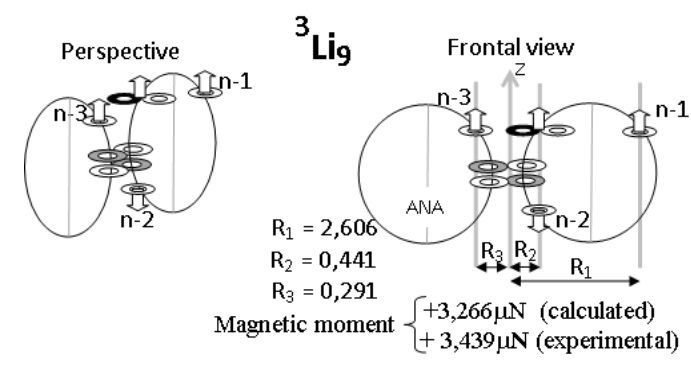

Fig 26. Structure of $3 L i 9$

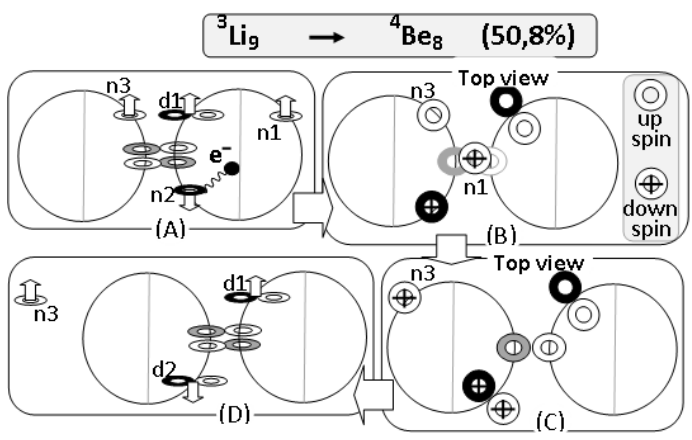

Fig 27. First decay mode of $3 \mathrm{Li} 9$

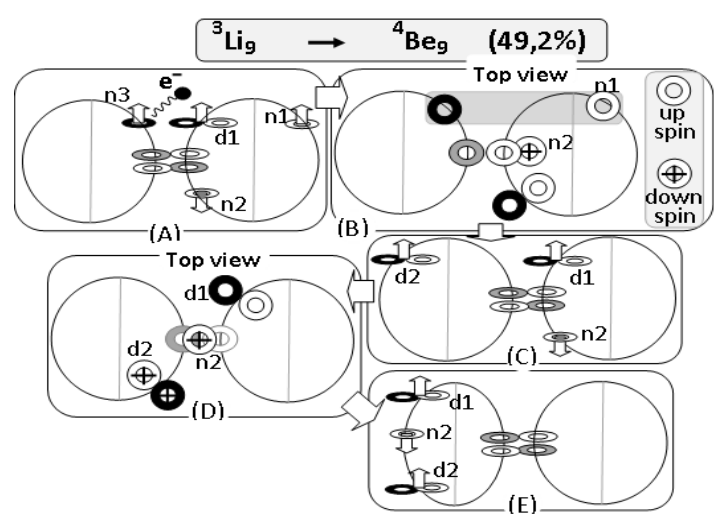

Fig 28. Second decay mode of $3 L i 9$

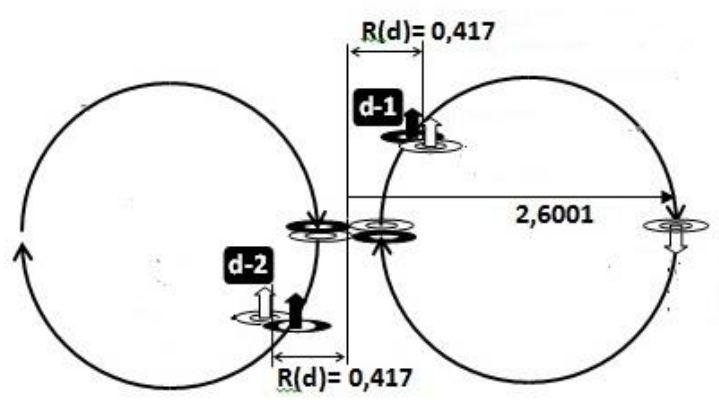

Fig 29. Structure of $4 B e 9$, whose magnetic moment is calculated in Part V. Note that the symmetry between the two deuterons is broken by the neutron, which avoids the two deuterons to fall down toward the central $2 \mathrm{He} 4$ (as occurs in $4 \mathrm{Be} 8$ ) 
Figure 30 compares the electric quadrupole moments of the nuclei $L i 6, L i 7, L i 9, L i 11$, and $B e 9$. Let us begin the explanation with Li6. The center of the central $2 \mathrm{He} 4$ has coordinates $(x, y, z)$, being $x=y=z=0$. In Li6 the charge of the deuteron is situated in a point with coordinates $(x, y, z)$, being $x=z=$ 0.417 , and $y=0$. So, the gray area in Figure 30 is a square with sides $x=0.417 \mathrm{fm}$ and $z=h=0.417 \mathrm{fm}$, and, due to the rotation of the nucleus about the z-axis, such square generates a spherical charge distribution, and so $Q($ Li6 $)=0$ ). However, there is an unbalance of mass due to the distance $\mathrm{x}$ of the deuteron to the $\mathrm{z}$-axis, and thereby the rotation of 3 Li6produces a shaking about the $\mathrm{z}$-axis, in order that the square becomes a rectangle with sides $\mathrm{h}=0.417$ and $W_{L i 6}=$ $0.355+\Delta_{L i 6}$. The rotation of the rectangle, about the $\mathrm{z}$-axis, produces a distribution of charge elongated along xy-plane (negative Q). The mass of the deuteron is relatively small regarding the total mass of the 3 Li6, and $\mathrm{x}=0.417$ is relatively short, in order that the unbalance in Li6 is weak, giving a short value for $\Delta_{L i 6}$. This is the reason why $Q(L i 6)$ is very small, $Q(L i 6)=-0.0008 b$, a value near to zero. Figure 30 shows that $\mathrm{Li7}$ has a strong unbalance of masses, because the radius of the deuteron orbit is $\sim 0.45 \mathrm{fm}$ (a little larger than that in Li6), but mainly because the radius of the neutron orbit is $2.391 \mathrm{fm}$. Such unbalance yields a large $\Delta_{L i 7}$, resulting in a large negative $Q(L i 7)=-0.04 b$. In $L i 9, h=0.441 \mathrm{fm}$, larger than $h=0.404 \mathrm{fm}$ in $L i 7$, a difference of $0.036 \mathrm{fm}$. So, in order to have $Q(L i 9)=$ $Q(L i 7)$, there is need to have at least $\Delta_{L i 9}=\Delta_{L i 7}+0.036 / 2$. But in $L i 9$ the inner neutron $\left(R_{3}=0.291 \mathrm{fm}\right)$ contributes for diminishing the unbalance, by producing a shaking in the contrary direction, in order that the shaking of $L i 9$ is only a little stronger than in $L i 7$, and that's why $Q(L i 9)=0.025<$ $Q(L i 7)=0.040$ (not considering the signs). $L i 11$ and $L i 9$ have values of Q very near, respectively $-0.031 b$ and $-0.025 b$, and let us understand why. While Li11 has $h=0.9$, Li9 has $h=$ 0.441 , and so, in order to have $Q(L i 11)=Q(L i 9)$, there is need to have $\Delta_{L i 11} \gg \Delta_{L i 9}$. And $\Delta_{L i 11}$ is indeed to be very larger than $\Delta_{L i 9}$, because $L i 11$ has unbalance of masses very stronger than $L i 9$, since $L i 11$ has two neutrons with $R=2.78 \mathrm{fm}$, while $L i 9$ has one neutron with $R=2.606 \mathrm{fm}$.

$B e 9$ has $Q>0$, because $h>w$, and the reason is seen in the Figure 30; the neutron causes a strong shaking of the $B e 9$ around the z-axis, but " $h$ " continues being larger than " $W_{B e} 9$ ".

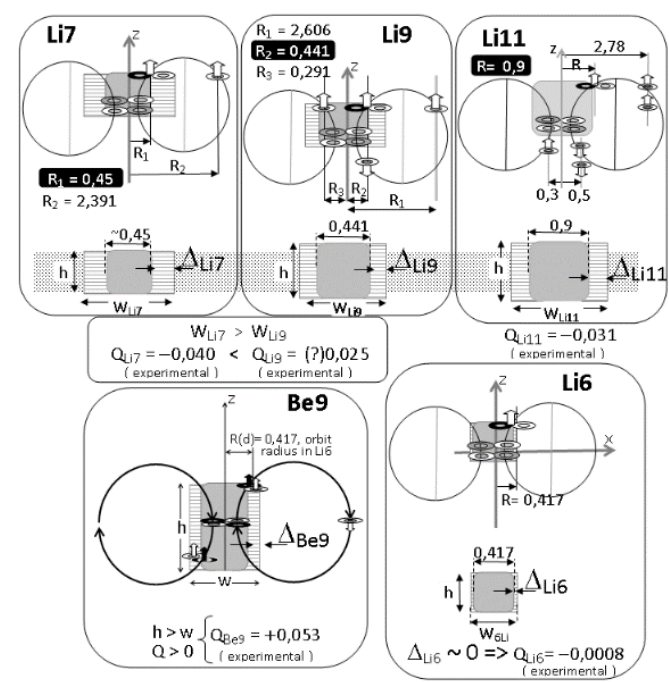

Fig 30. Influence of the unbalance of masses in the charge distribution into light nuclei
In the case of $\mathrm{Be} 7$ (shown in Figure 19), the distribution of charges has rectangular shape, with height " $h$ " and width " $L$ ", being $\mathrm{h}$ a little longer than $\mathrm{L}, h=L+\phi$. The unbalance of masses, due to the proton and deuteron, is weak, because their orbit radii are very short, in order that we may expect that $\Delta_{B e 7}=\phi / 2$. So, $\mathrm{h}$ and $W_{B e 7}$ have approximately the same length, and thereby $Q(B e 7)$ is very near to zero, as happens with $Q($ Li6).

\section{Li11 DECAY MODES}

Figure 31 shows the structure of Li11, and we realize that it is a good candidate of having several decay modes. From that structure the calculation of magnetic moment gives $\mu=$ $+3.749 \mu \mathrm{N}$, whereas the experimental value is $\mu=3.668 \mu \mathrm{N}$ (the sign is not quoted in nuclear tables).

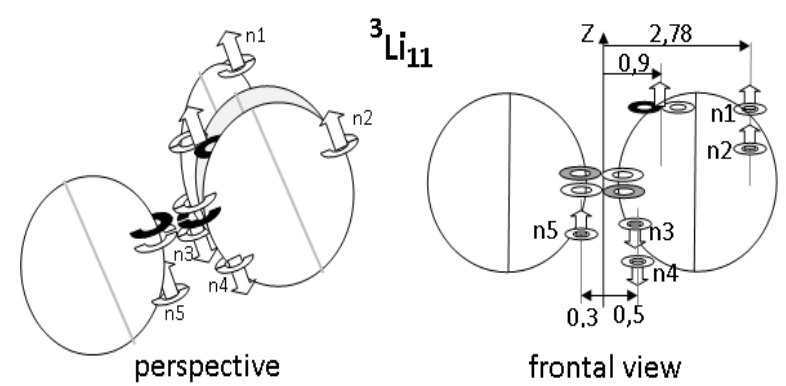

Fig 31. Structure of 3Li11: from the distribution of neutrons seen in perspective, we realize that 3 Li11 is a good candidate of having several decay modes.

3 Li11 decay modes are,

$B e_{10} \rightarrow \beta^{-}, n, 84.9 \% \quad ; B e_{11} \rightarrow \beta^{-}, 8.07 \% \quad B e_{9} \rightarrow \beta^{-}, 2 n, 4.1 \%$ $; \mathrm{Be}_{8} \rightarrow \beta^{-}, 3 \mathrm{n}, 1.9 \% \quad ; \quad\left(\mathrm{He}_{7}, \mathrm{He}_{4}\right) \rightarrow \beta^{-}, \alpha, 1.0 \% \quad ;\left(\mathrm{Li}_{8}, \mathrm{H}_{3}\right) \rightarrow$ $\beta^{-}$, fusion, $0.014 \% ;\left(\mathrm{Li}_{9}, \mathrm{H}_{2}\right) \rightarrow \beta^{-}$, fusion, $0.013 \%$

Unfortunately, the present paper is already too much long, in order that the sequence of positions and interactions of nucleons, step by step in each of the decay models, will not be presented here.

\section{CONCLUSIONS}

We cannot discover all the fundamental laws of nature via mathematics, because fundamental laws are consequence of physical mechanisms, whose some laws must be discovered either by experiments or by intuition. For instance, Maxwell has discovered his equations inspired by the experiments made by Faraday. Maxwell would never discover them via pure mathematics development. Nowadays the theorists suppose that fundamental laws, in a very deeper level than that discovered by Faraday, can be discovered by pure mathematical development. Nevertheless, in a deeper level there is need to consider the influence of the aether, and this is the reason why the attempt of discovering fundamental laws, via pure mathematics, sometimes requires the adoption of ad hoc mathematical assumptions, as for instance the asymptotic freedom. David Gross, Frank Wilczek, and David Politzer, had proposed asymptotic freedom in order to solve a fundamental puzzle, which however can be solved by the adoption of a new fundamental law, as proposed in (Guglinski, 2018b), where it's shown that, by considering a model of electric field composed by electricitons of the aether, the closer are two electric particles (whose charges have the same sign) the less 
the Coulomb repulsion is between them. While asymptotic freedom is actually a pseudoscientific theory, because it cannot be proven or disproven by experiments. The theory was accepted with open arms by the physicists because, as according to QED the Coulomb's law is invariable, then there was need to find the reason why quarks can be bound. As the puzzle was originated from a mathematical requirement, which origin lies in the assumption that Coulomb's law is invariable along any value of " $d$ ", then the solution had to be found via mathematics, as proposed by Gross-WilczekPolitzer. The proposal that Coulomb's law is actually $F=$ $K\left(Q q / d^{1 / x}\right)$, proposed in (Guglinski, 2018b), was considered too speculative by the editor of the International Journal of Modern Physics, and he has declined the paper. However, the new version for Coulomb's law can be proven, or disproved, by experiments, while the asymptotic freedom can never be tested by experiments. And so, what among the two proposals is the most speculative? In 1887 an experiment had invalidated the hypothesis that light is a propagation of electromagnetic disturbance in the aether. The experiment did not detect the aether, and Einstein faced a hard dilemma, as either changing the Maxwell equations, or changing the Galileo transformations. In order to find a solution, he had concluded that he could not any longer be loyal to the Newton's famous phrase, I shall not mingle conjectures with certainties. And Einstein decided to sacrifice Galileo. However, the immolation of Galileo was not enough, because nowadays new experiments are suggesting that Maxwell equations can be incomplete, and what is missing in his equations obviously has reflection in QED, as shown in several papers (Cruz, 2016; Cláudio Nassif, 2008; Claudio Nassif, 2010; Cláudio Nassif, 2012, 2015). Curiously Einstein had interpreted wrongly the meaning of the negative result in the Michelson-Morley experiment, because at that time he and the theorists had supposed that the nature of the light is undulatory, i.e., that light is a wave moving in the aether, and such supposal requires that Michelson's interferometer would have to detect a difference in his experiment. Nevertheless, the true meaning of the negative result of the experiment is that light is not wave, as supposed Einstein and his contemporaries. The negative result of the experiment does not deny the aether, what it actually denies is the undulatory nature of the light. Therefore, the aether was unduly denied and rejected along one century, because Einstein and the physicists interpreted wrongly the negative result of the Michelson-Morley experiment. Nowadays we have not only one experiment invalidating the untouchable foundations of the pillars of Modern Physics, as the Standard Nuclear Physics, the Standard Model, and the Quantum Electrodynamics. There are a lot of experiments invalidating them. The last one was published in August 13, 2016, when the deuteron was detected so smaller than expected (Pohl et al., 2016). Mirroring the proton radius puzzle, the radius of the deuteron was several standard deviations smaller than the value inferred from previous

\section{REFERENCES}

Antonello, M., Aprili, P., Baiboussinov, B., Ceolin, M. B., Benetti, P., Calligarich, E., Cieślik, K. (2012). Measurement of the neutrino velocity with the ICARUS detector at the CNGS beam. Physics Letters B, 713(1), 17-22. spectroscopic measurements of electronic deuterium. But the SM tells us that the interaction between a proton and muon should be identical to that between a proton and an electron. The dilemma today is worse than that faced by Einstein. The theorists must decide what they have to sacrifice. Many are proposing a new fifth force, in order to solve the puzzle of the proton radius, and others are proposing a fifth force in order to solve the puzzle of the pear shape of Ra224, and others are proposing a fifth force so that to explain the puzzle of the anomaly in the beryllium nuclear decays mediated by the mysterious boson X (Krasznahorkay et al., 2016). In this way, if will be need a new fifth force every time when a new puzzle debunks the SM and the QED, we will need not a new fifth particle, but a sixth, a seventh, a eighth, ... a twentieth... and so on, in order that each one of the new fundamental force will be applied so that to solve a specific puzzle, because, of course, will be impossible to connect all the anomalies to an unique force, in spite of, at the first glance, it may seems that is easy to solve any new puzzle by simply proposing a new fifth force. Besides, even the proposal of a new fifth force does not bring answers for the puzzles concerning the mysterious neutrino. André de Gouvêa, a theoretical physicist at Northwestern University, deems neutrinos the "only palpable evidence of physics beyond the standard model", and the theorists think that everything we learn about neutrinos in the coming years is new physics. They have now six reasons to believe that neutrinos might provide the window into new physics that the LHC has not, as

1- Neutrinos are proof that the standard model is wrong

2- Neutrinos' ability to morph from one flavor to another is only now starting to be understood

3- Neutrinos may exhibit charge conjugation-parity $(\boldsymbol{C P})$ violation

4- Neutrinos may be the first fundamental particles that are Majorana fermions

5- Another neutrino flavor may be waiting to be discovered 6- Multiple powerful neutrino experiments are on the horizon Obviously a seventh reason is missing, because

7- Neutrinos move with the speed of light, or even faster, and they violate the Lorentz's Factor

But the worst is that theorists have not the minimum idea on from "where" they have to start the new physics. Therefore, it's out of any doubt that the situation is very serious, because this panorama of crisis, which some theorists are calling "the LHC nightmare scenario", requires speculations beyond the proposal of a fifth force or the discovery of a new particle, with the hope of saving the foundations of the SM and the own QED. The pillars of the SM and QED are already demolished by the several new experimental finding published in the last decade. What is missing in the SM and QED is foundation piles for the pillars on which they were erected. The whole structure collapsed. It is necessary to erect New Pillars, having the aether as foundation piles.

Bahcall, J. (1998). Solar neutrinos: Where we are, what we need. Nuclear Physics A, 631, 29-41.

Cruz, C. N. (2016). On the electrodynamics of moving particles in a quasi flat spacetime with Lorentz violation and its cosmological implications. International Journal of Modern Physics D, 25(10), 1650096. 
Csótó, A., \& Langanke, K. (1998). Effects of 8B size on the low-energy $7 \mathrm{Be}(\mathrm{p}, \gamma) 8 \mathrm{~B}$ cross section. Nuclear Physics A, 636(2), 240-246.

Csótó, A., Langanke, K., Koonin, S., \& Shoppa, T. (1995). ^ $7 \mathrm{Be}(\mathrm{p}, \gamma)^{\wedge} 8 \mathrm{~B}$ cross section and the properties of ${ }^{\wedge} 7 \mathrm{Be}$ Physical Review C, 52(2), 1130-1133.

Ebran, J.-P., Khan, E., Nikšić, T., \& Vretenar, D. (2012). How atomic nuclei cluster. Nature, 487(7407), 341.

Gaffney, L. P., Butler, P. A., Scheck, M., Hayes, A. B., Wenander, F., Albers, M., Bönig, S. (2013). Studies of pearshaped nuclei using accelerated radioactive beams. Nature, 497(7448), 199.

Guglinski, W. (2006). Quantum Ring Theory, : Bäuu Institute Press. Boulder, Co, US.

Guglinski, W. (2018a). Calculation of proton radius to be measured in the Project MUSE. Physics Essays, 31(2), 137.

Guglinski, W. (2018b). On how Bohr model of hydrogen atom is connected to nuclear physics. International Journal of Fundamental Physical Sciences, 8(2), 45-54. doi: https://doi.org/https://doi.org/10.14331/ijfps.2018.330113

Guglinski, W. (2018c). Re-evaluation of Fermi's theory of beta-decay. International Journal of Fundamental Physical Sciences, 8(2), 19-43.

Krasznahorkay, A., Csatlós, M., Csige, L., Gácsi, Z., Gulyás, J., Hunyadi, M., Timár, J. (2016). Observation of Anomalous Internal Pair Creation in Be 8: A Possible Indication of a Light, Neutral Boson. Physical review letters, 116(4), 042501 .
Krieger, A., Blaum, K., Bissell, M. L., Frömmgen, N., Geppert, C., Hammen, M., .Neff, T. (2012). Nuclear Charge Radius of Be 12. Physical review letters, 108(14), 142501.

Nassif, C. (2008). Deformed special relativity with an invariant minimum speed and its cosmological implications. Pramana, 71(1), 1-13.

Nassif, C. (2010). Deformed Special Relativity with an energy barrier of a minimum speed. International Journal of Modern Physics D, 19(05), 539-564.

Nassif, C. (2012). Double special relativity with a minimum speed and the uncertainty principle. International Journal of Modern Physics D, 21(02), 1250010.

Nassif, C. (2015). An explanation for the tiny value of the cosmological constant and the low vacuum energy density. General Relativity and Gravitation, 47(9), 107.

Nörtershäuser, W., Tiedemann, D., Žáková, M., Andjelkovic, Z., Blaum, K., Bissell, M., Kowalska, M. (2009). Nuclear Charge Radii of Be 7, 9, 10 and the One-Neutron Halo Nucleus Be 11. Physical review letters, 102(6), 062503.

Pohl, R., Antognini, A., Nez, F., Amaro, F. D., Biraben, F., Cardoso, J. M., . Fernandes, L. M. (2010). The size of the proton. Nature, 466(7303), 213.

Pohl, R., Nez, F., Fernandes, L. M., Amaro, F. D., Biraben, F., Cardoso, J. M., . Diepold, M. (2016). Laser spectroscopy of muonic deuterium. Science, 353(6300), 669-673.

Tang, Z. (2018). Search for the Neutron Decay $n \rightarrow X+\gamma$ where $\mathrm{X}$ is a dark matter particle. arXiv preprint arXiv:1802.01595. 